\title{
Integrated experimental-computational analysis of a liver-islet microphysiological system for human-centric diabetes research
}

Belén Casas ${ }^{1,2}$ Liisa Vilén ${ }^{1}$, Sophie Bauer ${ }^{3}$, Kajsa Kanebratt ${ }^{1}$, Charlotte Wennberg Huldt ${ }^{4}$, Lisa Magnusson $^{4}$, Uwe Marx ${ }^{3}$, Tommy B. Andersson ${ }^{1}$, Peter Gennemark ${ }^{1,2, \dagger}$ Gunnar Cedersund $^{2,5, *, \dagger}$

1 Drug Metabolism and Pharmacokinetics, Research and Early Development, Cardiovascular, Renal and Metabolism (CVRM), BioPharmaceuticals R\&D, AstraZeneca, Gothenburg, Sweden.

2 Department of Biomedical Engineering, Linköping University, Linköping, Sweden.

3 TissUse GmbH, Berlin, Germany.

4 Bioscience, Research and Early Development, Cardiovascular, Renal and Metabolism (CVRM),

BioPharmaceuticals R\&D, AstraZeneca, Gothenburg, Sweden.

5 Center for Medical Image Science and Visualization (CMIV), Linköping University, Linköping, Sweden.

$\dagger$ Shared senior author

*Corresponding author

Gunnar Cedersund, Department of Biomedical Engineering

Linköping University,

SE-581 83 Linköping, Sweden.

E-mail: gunnar.cedersund@liu.se 


\section{Abstract}

Microphysiological systems (MPS) are powerful tools for emulating human physiology and replicating disease progression in vitro. MPS could be better predictors of human outcome than current animal models, but mechanistic interpretation and in vivo extrapolation of the experimental results remain significant challenges. Here, we address these challenges using an integrated experimentalcomputational approach. This approach allows for in silico representation and predictions of glucose metabolism in a previously reported MPS with two organ compartments (liver and pancreas) connected in a closed loop with circulating medium. We developed a computational model describing glucose metabolism over 15 days of culture in the MPS. The model was calibrated on an experiment-specific basis using data from seven experiments, where single-liver or liver-islet cultures were exposed to both normal and hyperglycemic conditions resembling high blood glucose levels in diabetes. The calibrated models reproduced the fast (i.e. hourly) variations in glucose and insulin observed in the MPS experiments, as well as the long-term (i.e. over weeks) decline in both glucose tolerance and insulin secretion. We also investigated the behavior of the system under hypoglycemia by simulating this condition in silico, and the model could correctly predict the glucose and insulin responses measured in new MPS experiments. Last, we used the computational model to translate the experimental results to humans, showing good agreement with published data of the glucose response to a meal in healthy subjects. The integrated experimental-computational framework opens new avenues for future investigations toward disease mechanisms and the development of new therapies for metabolic disorders.

Keywords: In silico modelling, In vitro to in vivo extrapolation, Type 2 diabetes mellitus, quantitative systems pharmacology, organ-on-a-chip 


\section{Introduction}

Type 2 diabetes mellitus (T2DM) is a complex multifactorial disease characterized by impaired glucose homeostasis. In healthy individuals, plasma glucose levels are maintained within a narrow range (3-9 $\mathrm{mM}$ ) (Balducci, 2014) via a negative feedback between glucose and insulin. Insulin is secreted in response to elevated glucose levels, increasing glucose uptake in target tissues (adipose, muscle and liver) to restore normoglycemia (Bergman, 1989). In early stages of the development into T2DM, target tissues become insulin resistant and require higher insulin concentrations to maintain normal glucose levels (Petersen and Shulman, 2018). Initially, $\beta$ cells compensate for insulin resistance through upregulation of insulin secretion ( $\beta$-cell adaptation), but over time they are unable to meet the increased insulin demand and overt T2DM manifests (Stumvoll et al., 2005). While these general steps in the disease etiology are well established, more detailed knowledge of the interplay between insulin resistance, pancreatic $\beta$-cell adaptation, and the progression of T2DM is still missing.

Currently, research on the pathogenesis and potential therapeutic agents in T2DM is primarily based on in vivo animal models (Calcutt et al., 2009). However, the translatability of these animal-based studies to human outcome is often limited. One fundamental obstacle for this translation is the naturally existing phylogenetic difference between the animals typically used in preclinical testing and humans. While these models can still be valuable for mechanistic and mode of action studies, the majority of drug candidates that show promise in preclinical animal studies ultimately fail to result in functional and safe drugs in humans (Olson et al., 2000; Chandrasekera and Pippin, 2014).

Because of aforementioned limitations in using animal studies, there is a critical need for novel preclinical models that can better represent human physiology and predict in vivo outcomes. This need has fueled the development of microphysiological systems (MPS), which are microscale devices capable of replicating human physiology in vitro. By integrating $3 \mathrm{D}$ cultures of human organ-specific cells in a microfluidic platform, these in vitro systems aim to recreate key microenvironmental aspects of in vivo tissues (flow, multicellular architectures, and tissue-tissue interfaces), thereby being more physiologically relevant than standard cell cultures (Esch et al., 2015; Marx et al., 2016; Zhang et al., 2018).

We have previously presented a two-organ MPS integrating liver and pancreas, which offers an advantage over single-organ MPS for studying glucose homeostasis (Bauer et al., 2017). Recent advances in MPS technology have led to the development of single organ-MPS for both liver (Lee et al., 2007; Materne et al., 2013; Banaeiyan et al., 2017; Du et al., 2017; Ortega-Prieto et al., 2018) and pancreas (Wu Jin et al., 2021), which are two major organs involved in the maintenance of glucose homeostasis. However, single organ-MPS have limited relevance for studying metabolic diseases like T2DM, as the underlying pathophysiology involves disruption in the homeostatic cross-talk between 
several organs. Therefore, multi-organ platforms capable of capturing interactions between two or more organs are best suited for investigating these diseases in vitro. Our previously developed liver-pancreas MPS supports a homeostatic feedback loop between co-cultured human liver spheroids and pancreatic islets (Bauer et al., 2017). This MPS allows detailed investigations of mechanisms underlying T2DM through enabling changes in both the operating and co-culture conditions in a controlled and systematic manner. For instance, one could perform changes in the glycemic levels or the composition of the coculture medium, as well as in the number and metabolic functions of the co-cultured cells. Moreover, unlike other in vivo and in vitro models, this experimental setup offers great flexibility to study interactions between specific subsets of organs. Therefore, assays based on this system could become superior to animal experiments for studying disease progression and drug metabolism.

However, to improve the applicability of our liver-pancreas MPS, two major challenges should be addressed. First, there is still limited mechanistic understanding of the physiological processes in the MPS. Elucidating these mechanisms using a purely experimental approach would be challenging, as the biological processes underlying glucose-insulin regulation in the MPS are complex, non-linear, and involve numerous feedback loops. Because of these complexities, relying on qualitative analysis and statistics of the experimental data may often lead to incorrect conclusions (Jullesson et al., 2015; Nyman et al., 2015). Second, the experimental findings from the system cannot be directly extrapolated to in vivo, human outcome. Although existing strategies for on-platform scaling could be applied to achieve in vitro responses that better mimic those observed in vivo, these have proven insufficient to ultimately establish the translation to humans (Cirit and Stokes, 2018). These two challenges could be confronted using computational modelling. More specifically, computational modelling provides a framework to quantitatively represent the system including its nonlinearities and feedback loops, integrate and interpret the experimental measurements, infer physiological variables that cannot be directly measured in vitro, and enable in vitro to in vivo translation.

While several studies have shown the added value of combining computational models with multiorgan MPS for data interpretation, these have mainly focused on pharmacokinetic (PK) (Prot et al., 2014; Kimura et al., 2015; Edington et al., 2017; Lee, Ha, et al., 2017) and in some cases pharmacokinetic/pharmacodynamic (PKPD) strategies (Sung et al., 2010; McAleer et al., 2019; Novak et al., 2020), rather than providing mechanistic understanding of the underlying physiology. Some efforts have been done to integrate MPS with more descriptive models, often referred to as quantitative systems pharmacology (QSP) models (van der Graaf and Benson, 2011;Cirit and Stokes, 2018). These models generally incorporate physiological parameters describing the MPS operating conditions, such as organoid sizes and flow rates, and more mechanistic knowledge about the physiology of the system. However, to date, the number of studies exploring this approach is still limited (Stokes et al., 2018; Maass et al., 2019; Yu et al., 2015), especially for studying glucose metabolism. A few studies have also 
focused on computational strategies to extrapolate the in vitro responses to human proportions. Typically, these strategies account for constraints in the in vitro setting that limit the capability of the MPS to reproduce human-like responses. These constraints generally include sizes of the organoids and the co-culture media, mismatches in media-to-tissue ratio and the fact that some organs and functions are missing in the MPS (Stokes et al., 2015; Sung et al., 2019). In a recent study, Lee et al. (Lee et al., 2019) presented a computational model for a pancreas-muscle MPS to study glucose metabolism, and added a liver compartment in silico to improve the physiological relevance of glucose and insulin dynamics in the system. However, the model was constructed using experimental data from individual cultures of myoblasts and pancreatic cells from rodents, and did not incorporate measurements from interconnected co-cultures that could reflect organ cross-talk. Despite the crucial role of the liverpancreas cross-talk in maintaining glucose homeostasis, the combination of a mechanistic computational model and an MPS emulating the interaction between these organs has not been investigated yet.

In this study, we propose combining our liver-pancreas MPS with a computational model to augment in vitro investigations of human glucose homeostasis in healthy and hyperglycemic conditions mimicking high blood glucose levels in T2DM. Our aim is to use the model to integrate and quantitatively analyze the experimental data to improve their mechanistic interpretation, generate model predictions and, ultimately, extrapolate the results from in vitro to in vivo. The possibility to generate human-relevant predictions from in vitro experiments at a relatively low cost could reduce the need for animal models of T2DM in the future.

\section{Materials and Methods}

\subsection{In vitro experiments}

To construct, calibrate and evaluate the computational model we used data from seven independent in vitro MPS experiments (Supplementary Table S1). Two of the experiments (experiments 1 and 2) have already been published in (Bauer et al., 2017). In the following section, we describe the materials and methods for the five experiments performed for this study.

\subsubsection{Multi-organ chip platform}

To co-culture liver spheroids and pancreatic islet microtissues (from now on referred to as pancreatic islets), we used the Chip2 from TissUse which allows for simultaneous culture of two organ models in spatially separated, but interconnected culture compartments (Fig. 1A,B). Details on the design and fabrication process of the Chip2 are described in prior publications (Schimek et al., 2013; Wagner et al., 2013). The culture compartments are connected by a microfluidic channel, with an on-chip micropump driving a continuous pulsatile flow that supports long-term perfusion of the chip-cultured 3D cell constructs. Both culture compartments contain $300 \mu \mathrm{L}$ of culture medium and the microfluidic channels 
hold additional $5 \mu \mathrm{L}$ of medium. The average volumetric flow rate between the compartments was set to $4.94 \mu \mathrm{L} / \mathrm{min}$, resulting in an approximate medium turnover time of $2 \mathrm{~h}$.

\subsubsection{Pre-culture of liver spheroids and pancreatic islets}

Human liver spheroids were formed in 384-well spheroid microplates (3830, Corning) by combining differentiated pre-cultured HepaRG cells (Lot HPR116189, HPR116239, HPR116246, HPR116NS080003 or HPR116222; Biopredic International; France) and passaged primary human hepatic stellate cells (Lot PFP; BioreclamationIVT; USA) at a ratio of 24:1 as previously described (Bauer et al 2017). HepaRG pre-cultures and liver spheroids were maintained in Williams medium $\mathrm{E}$ supplemented with $10 \%$ FBS, $2 \mathrm{mM}$ L-glutamine, $50 \mu \mathrm{M}$ hydrocortisone hemisuccinate, $50 \mu \mathrm{g} / \mathrm{ml}$ gentamycin sulfate and $0.25 \mu \mathrm{g} / \mathrm{ml}$ amphotericin B. Glucose and insulin concentrations in the HepaRG maintenance medium were set according to the glycemic level used in the MPS culture. For cultures in hyperglycemia, normoglycemia or hypoglycemia, the pre-culture medium contained $11 \mathrm{mM}$ glucose and $860 \mathrm{nM}$ insulin, $5.5 \mathrm{mM}$ glucose and $1 \mathrm{nM}$ insulin, or $2.8 \mathrm{mM}$ glucose and $0.1 \mathrm{nM}$ insulin, respectively. The medium used for the pre-cultured HepaRG cells was additionally supplemented with $2 \%$ DMSO, while this was omitted in the medium for spheroid formation in order to avoid harmful effects on the human hepatic stellate cells. Human pancreatic islet microtissues were purchased from InSphero (MT04-002-0; Switzerland) and maintained in 3D InSight ${ }^{\mathrm{TM}}$ Human Islet Maintenance Medium (CS-07005-02; InSphero) until the MPS culture. The pancreatic islet donors included five men, with an age of $54 \pm 5$ years (range $45-57$ years), body mass index (BMI) $28 \pm 2 \mathrm{~kg} / \mathrm{m}^{2}$ (range 27-30 kg/m²), hemoglobin A1c (HbA1c) $5.6 \pm 0.3 \%$ (range 5.1-5.8\%) with no known history of diabetes. All cell cultures were maintained at $37{ }^{\circ} \mathrm{C}$ and $5 \% \mathrm{CO}_{2}$.

\subsubsection{MPS cultures}

Before tranferring liver spheroids and pancreatic islets into the Chip2, both were washed twice with $0.1 \%$ BSA in 1xPBS and pre-incubated in insulin-free HepaRG maintenance medium for 2 hours. To setup the co-culture, 40 liver spheroids and 10 pancreatic islets were placed into the liver and pancreas compartments, respectively. In comparison to their respective human counterparts, this corresponds to a downscaling factor of 100,000 in both organs (Wilson et al., 2003; Narang and Mahato, 2006). In single-liver cultures, 40 liver spheroids were added into the liver compartment while keeping the pancreas compartment empty. Both the co-cultures and single-organ cultures were maintained in insulinfree HepaRG medium (referred as chip medium from here on) with glucose concentration of $11 \mathrm{mM}$, $5.5 \mathrm{mM}$ or $2.8 \mathrm{mM}$ depending on the glycemic regime (Figure 1C). The chip culture medium with respective glucose level was changed first after 24 hours and then after every 48 hours over the culture period of 15 days. In each individual experiment, all glycemic levels were studied in 4-10 replicates. 


\subsubsection{In vitro glucose tolerance test}

Regulation of glucose homeostasis as a result of organ cross-talk was assessed by in vitro glucose tolerance tests (GTT) (Bauer et al. 2017). On day 13, $315 \mu 1$ of co-culture medium with $11 \mathrm{mM}$ glucose was added into each of the two culture compartments and samples of $15 \mu 1$ were collected after $0,8,24$ and 48 hours. The samples collected from both compartments were pooled together for glucose and insulin analysis, resulting in a maximal volume decrease of $10 \%$ over the entire GTT. Additionally, a similar sampling scheme was performed on day 1 but keeping the respective glycemic level as indicated for each regime.

\subsubsection{Glucose and insulin analysis}

Glucose concentrations were determined either using the GLU 142 kit (Diaglobal, Berlin, Germany) as described previously (Bauer et al. 2017) or using Glucose Liquid Reagent (1070-400, Stanbio) with minor modifications to the manufacturer's instructions. Briefly, $5 \mu 1$ of culture supernatant was mixed with $95 \mu 1$ of pre-heated assay reagent and after 5 min incubation at $37{ }^{\circ} \mathrm{C}$ degrees the absorbance was measured at $520 \mathrm{~nm}$. Insulin concentrations were measure using Insulin ELISA (10-1113-01, Mercodia) following the manufacturer's instructions.

\subsubsection{Glucose-stimulated insulin secretion}

After the MPS culture, pancreatic islets were transferred from the chips into a GravityTRAP ${ }^{\mathrm{TM}}$ plate (InSphero) to analyse their glucose-stimulated insulin secretion (GSIS) as earlier described (Bauer et al 2017). In brief, pancreatic islets were equilibrated in low glucose buffer $(2.8 \mathrm{mM})$ for $2 \mathrm{~h}$ followed by sequential $2 \mathrm{~h}$ incubations first in low glucose buffer and then in high glucose buffer (16.8 $\mathrm{mM})$.

\subsection{Computational model of the liver-islet MPS}

We developed a computational model describing glucose metabolism in the liver-islet MPS. The model is based on data from seven independent experiments corresponding to seven different donors of pancreatic islets $(\mathrm{N}=7)$. The model, outlined in Figure 2, describes key physiological processes underlying glucose regulation on a short-term (meal response) basis and the long-term changes in insulin resistance and $\beta$-cell adaptation associated with impaired glucose homeostasis. We included two model compartments, each of them representing a specific organoid (liver or pancreas) and its corresponding co-culture medium. The compartments are connected in a closed loop, and the medium circulates as specified by a flow rate parameter.

The model is based on the long-term glucose, insulin and $\beta$-cell mass dynamics proposed by Topp et al. (Topp et al., 2000). Here, we have modified this model to: 1) encompass two model components with different time scales: a fast (hours) component for glucose and insulin dynamics between media exchanges, and a slow (weeks) component describing the development of hepatic insulin resistance and 
$\beta$-cell adaptation, 2) explicitly establish an interaction between the fast and slow model components, allowing short-term dynamics of physiological variables to impact long-term progression of the disease (e.g. effects of daily glucose levels on insulin resistance and $\beta$-cell volume dynamics), 3) support scaling to humans by specifying organ sizes and operating conditions in the MPS (i.e. flow rate between culture compartments and co-culture media volumes) and 4) allow inhibition in $\beta$-cell insulin secretion over time.

The model was formulated using a system of mass-balanced, non-linear ordinary differential equations (ODEs), and well-mixed conditions were assumed in each compartment. A complete description of the model equations including definitions of the metabolic fluxes in the system is provided in the Supplementary material.

\subsubsection{Modelling short-term glucose homeostasis in the co-culture}

The short-term glucose homeostasis was modelled in the following way. First, we based our description of glucose on the glucose equation in the model of Topp et al. (Topp et al., 2000). This equation derives from a simplification of the minimal glucose model (Bergman et al., 1979) to represent daily average glucose (Ha et al., 2015). Glucose content in the liver compartment culture medium is controlled by glucose dosing to the system, endogenous glucose production and glucose uptake by the liver spheroids, as well as glucose inflow from and outflow to the pancreas compartment:

$$
\begin{aligned}
\frac{d N G_{m, \text { liver }}(t)}{d t}= & G_{d}(t)+V_{\text {HepaRG, spheroids }} \cdot \operatorname{EGP}(t) \\
& -V_{\text {HepaRG,spheroids }}\left(E_{G 0}+S_{I}(t) \cdot \frac{N I_{m, \text { liver }}(t)}{V_{m, \text { liver }}}\right) \frac{N G_{m, \text { liver }}(t)}{V_{m, \text { liver }}}+Q \cdot \frac{N G_{m, \text { pancreas }}(t)}{V_{m, \text { pancreas }}} \\
& -Q \cdot \frac{N G_{m, \text { liver }}(t)}{V_{m, \text { liver }}}(\mathrm{mmol} / \mathrm{h})
\end{aligned}
$$

where the symbols in the equation are defined as follows. $N G_{m, l i v e r}(t)$ and $N G_{m \text {,pancreas }}(t)$ are the number of glucose molecules (mmol) in the culture media corresponding to the liver and pancreas compartments, respectively, and $N I_{m, \text { liver }}(t)$ is the number of insulin molecules in the liver compartment's co-culture medium (mIU). The glucose input rate $G_{d}(t)(\mathrm{mmol} / \mathrm{h})$ represents glucose variations due to media exchanges, and $\operatorname{EGP}(t)$ describes endogenous glucose production in the liver spheroids $(\mathrm{mmol} / \mathrm{L} / \mathrm{h})$. After analysing the experimental data, $\operatorname{EGP}(t)$ was concluded to be negligible based on the observed decline in glucose levels below normoglycemia $(5.5 \mathrm{mM})$ in the system. Therefore, in practice, $\operatorname{EGP}(t)$ was set to zero. Glucose uptake by the liver spheroids is largely dependent on the insulin-independent glucose disposal rate (denoted $E_{G 0}(1 / \mathrm{h})$ ), but is also enhanced by the action of insulin. This enhancement accounts for an increased glucose influx through the GLUT2 hepatic transporter as a result of the reduction in intracellular glucose via insulin-induced metabolic pathways (e.g. glycogen synthesis and de novo lipogenesis) (König et al., 2012; Petersen and Shulman, 2018). The variable $S_{I}(t)(\mathrm{L} / \mathrm{mIU} / \mathrm{h})$ denotes the insulin sensitivity of the liver spheroids. The 
parameters representing operating conditions include the flow rate between culture compartments (denoted $Q(\mathrm{~L} / \mathrm{h})$ ), the total volume of HepaRG cells in the liver spheroids $\left(V_{\text {HeparG,spheroids }}(\mathrm{L})\right)$ and the volume of co-culture medium in the liver and pancreas compartments $\left(V_{m, l i v e r}\right.$ and $V_{m, \text { pancreas }}(\mathrm{L})$, respectively), as previously described by Lee et al. (Lee et al., 2019).

Second, the release of insulin from $\beta$-cells in the pancreatic islets was modelled as a sigmoidal function of glucose concentration (Malaisse et al., 1967; Topp et al., 2007; Ha et al., 2015):

$$
\begin{aligned}
\frac{d N I_{m, \text { pancreas }}(t)}{d t} & =V_{\beta, \text { islets }}(t) \cdot \sigma(t) \cdot \frac{\left(\frac{N G_{m, \text { pancreas }}(t)}{V_{m, \text { pancreas }}}\right)^{2}}{E C 50_{I}^{2}+\left(\frac{N G_{m, \text { pancreas }}(t)}{V_{\text {m,pancreas }}}\right)^{2}}+Q \frac{N I_{m, \text { liver }}(t)}{V_{m, l i v e r}} \\
& -Q \frac{N I_{m, \text { pancreas }}(t)}{V_{m, \text { pancreas }}}(\mathrm{mIU} / \mathrm{h})
\end{aligned}
$$

where $N I_{m \text {,pancreas }}(t)$ and $N I_{m, l i v e r}(t)$ are the number of insulin molecules $(\mathrm{mIU})$ in the pancreas and the liver compartment, respectively. Insulin secretion depends on the volume of $\beta$ cells in the pancreatic islets (denoted $V_{\beta, \text { islets }}(t)(\mathrm{L})$ ), the insulin secretion capacity per unit volume of $\beta$ cells (denoted $\sigma(t)$ $(\mathrm{mIU} / \mathrm{L} / \mathrm{h})$ ), and the glucose concentration resulting in half-of-maximum response to insulin (denoted $\left.E C 50_{I}(\mathrm{mmol} / \mathrm{L})\right)$. We account for a decrease in the insulin secretion capacity of the $\beta$ cells over time, as given by:

$$
\sigma(t)=\sigma_{\max } \cdot\left(1-\frac{t^{2}}{\alpha+t^{2}}\right)(m I U / L / h)
$$

Here, we assume that this decrease follows a sigmoidal dependence on time, determined by the parameter $\alpha\left(\mathrm{h}^{2}\right)$. The parameter $\sigma_{\max }(\mathrm{mIU} / \mathrm{L} / \mathrm{h})$ represents the maximal insulin secretion rate of the $\beta$ cells (i.e. at the beginning of the co-culture). The parameter $\alpha$ is estimated based on the experimental measurements. For large values of this parameter, the decrease in insulin secretion capacity over time would be negligible.

\subsubsection{Modelling hepatic insulin resistance and $\beta$-cell dynamics}

The dynamics of hepatic insulin sensitivity $S_{I}(t)$ were modelled under the assumption that insulin responsiveness of the co-cultured liver spheroids decreases because of sustained exposure to hyperglycemia: 


$$
\begin{aligned}
& S_{I}(t)=S_{I 0} \cdot\left(1-\frac{I_{\text {max }, S i} \cdot G_{\text {int }}(t)}{E C 50_{S i}+G_{\text {int }}(t)}\right)(L / m I U / h) \\
& \frac{d G_{\text {int }}(t)}{d t}=\left\{\begin{array}{ll}
\frac{N G_{m, l i v e r}(t)}{V_{m, \text { liver }}}-G_{\text {normo }} & \frac{N G_{m, \text { liver }}(t)}{V_{m, \text { liver }}}-\text { Gnormo } \geq 0 \\
0 & \frac{N G_{m, \text { liver }}(t)}{V_{m, \text { liver }}}-\text { Gnormo }<0
\end{array} \quad\left(\frac{m m o l}{L}\right)\right.
\end{aligned}
$$

where $S_{I 0}(\mathrm{~L} / \mathrm{mIU} / \mathrm{h})$ is the insulin sensitivity at the start of the co-culture. $S_{I}(t)$ decreases progressively as the liver spheroids are exposed to glucose levels above the normoglycemic range in the co-culture medium, which we refer to as excess glucose $\left(\frac{N G_{m, l i v e r}(t)}{V_{m, l i v e r}}-G\right.$ normo $\left.\geq 0\right) . G_{\text {int }}(t)$ represents the integral of excess glucose over time. The proposed model captures the hypothesis postulated by Bauer et al. (Bauer et al., 2017) that even short hyperglycemic periods $(<24 \mathrm{~h})$ could induce insulin resistance in the co-cultured liver spheroids, resulting in an increase in glucose levels over time as observed in our system. This is also consistent with the results from Davidson et al. (Davidson et al., 2016), which reported the development of insulin resistance in primary human hepatocytes after six days of exposure to a hyperglycemic culture medium containing $25 \mathrm{mM}$ glucose. In our model, the decrease in $S_{I}(t)$ was represented by a sigmoidal function with maximal fractional reduction $I_{\max , S i}$, and with half of the maximal fractional reduction occurring at $E C 50_{S i}(\mathrm{mmol} \cdot \mathrm{h} / \mathrm{L})$.

In the computational model, insulin secretion depends on both the total volume of $\beta$ cells in the pancreatic islets and their individual secretion capacity. The $\beta$ cells adapt to the long-term (slow) changes in glucose concentration by regulating their rates of replication and apoptosis, as previously described by Topp et al. (Topp et al., 2000). This adaptation changes the number of $\beta$ cells, with an associated change in total $\beta$-cell volume $\left(V_{\beta, \text { islets }}(t)\right)$ given by:

$$
\begin{gathered}
\frac{d V_{\beta, \text { islets }}(t)}{d t}=(\text { Replication }- \text { Apoptosis }) \cdot V_{\beta, \text { islets }}(t)(L / h) \\
\text { Replication }=k_{v} \cdot\left(r_{1, r} G_{\text {slow,pancreas }}(t)-r_{2, r} G_{\text {slow,pancreas }}(t)^{2}\right) \\
\text { Apoptosis }=k_{v} \cdot\left(d_{0}-r_{1, a} G_{\text {slow,pancreas }}(t)+r_{2, a} G_{\text {slow,pancreas }}(t)^{2}\right)
\end{gathered}
$$

where the rates of replication and apoptosis are modelled as nonlinear functions of glucose concentration in the medium, on the basis of previous in vitro studies (Chick, 1973; Swenne, 1982; Hoorens et al., 1996; Efanova et al., 1998). The parameter $d_{0}$ is the death rate at zero glucose $\left(\mathrm{h}^{-1}\right)$ and $r_{1, r}, r_{1, a}$ $(\mathrm{L} / \mathrm{mmol} / \mathrm{h}), r_{2, r}, r_{2, a}\left(\mathrm{~L}^{2} / \mathrm{mmol}^{2} / \mathrm{h}\right)$ are parameters that determine the dependence of the replication and apoptosis rates on glucose. The parameter $k_{v}$ was introduced to account for potential differences in behaviour between human pancreatic islets in our in vitro system and rodent islets in the model of Topp et al. (Topp et al., 2000). 
We have modified the original insulin secretion model (Topp et al., 2000) by introducing the variable $G_{\text {slow,pancreas }}(t)$, which represents the long-term average glucose concentration in the co-culture medium, as given by:

$$
\frac{d G_{\text {slow,pancreas }}(t)}{d t}=\frac{G_{\text {pancreas }}(t)-G_{\text {slow,pancreas }}(t)}{\tau_{\text {slow }}}(\mathrm{mmol} / \mathrm{L} / \mathrm{h})
$$

where $G_{\text {pancreas }}(t)$ is calculated from the number of glucose molecules in the co-culture medium corresponding to the islets compartment $N G_{m \text {,pancreas }}(t)(\mathrm{mmol})$ and $V_{m \text {,pancreas }}\left(G_{\text {pancreas }}(t)=\right.$ $N G_{m, \text { pancreas }}(t) / V_{\text {m,pancreas }}$ ) and $\tau_{\text {slow }}$ (h) is a time constant that determines the averaging of $G_{\text {pancreas }}(t)$ over time. Previous in vivo and in vitro studies on rodent pancreatic islets have demonstrated changes in $\beta$-cell mass and proliferation via glucose stimulation on a time scale of days (Chick, 1973; Swenne, 1982; Bonner-Weir et al., 1989; Bernard et al., 1998; Paris et al., 2003). Therefore, the value of $\tau_{\text {slow }}$ was chosen so that $G_{\text {slow,pancreas }}(t)$ represents daily average glucose in the co-culture medium.

The equation describing the dynamics of $\beta$-cell volume (Eq. 6) can then be rewritten as follows:

$$
\frac{d V_{\beta, \text { islets }}(t)}{d t}=k_{v}\left(-d_{0}+r_{1} G_{\text {slow,pancreas }}(t)-r_{2} G_{\text {slow,pancreas }}(t)^{2}\right) \cdot V_{\beta, \text { islets }}(t)(L / h)
$$

where $r_{1}=r_{1, r}+r_{1, a}(\mathrm{~L} / \mathrm{mmol} / \mathrm{h})$ and $r_{2}=r_{2, r}+r_{2, a}\left(\mathrm{~L}^{2} / \mathrm{mmol}^{2} / \mathrm{h}\right)$. The formulation for the rate of change of $\beta$-cell number $\left(k_{v}\left(-d_{0}+r_{1} G_{\text {slow,pancreas }}(t)-r_{2} G_{\text {slow,pancreas }}(t)^{2}\right)\right.$ captures the hypothesis that a small increase in glucose from normoglycemia (i.e. mild hyperglycemia) leads to an increase in total $\beta$-cell volume in order to restore glucose homeostasis, while a higher glucose concentration drives total $\beta$-cell volume down instead (Topp et al., 2000; Ha et al., 2015). Based on the study from Topp et al. (Topp et al., 2000), the values of $r_{1}$ and $r_{2}$ were chosen to achieve two steady state solutions at glucose concentrations corresponding to 5.55 and $13.87 \mathrm{mM}$, resulting in a net increase in $\beta$-cell volume when glucose levels are in the range $5.55-13.87 \mathrm{mM}$.

\subsection{Model calibration}

The model has a total of 24 parameters. All the model parameters and the method used to set their values are listed in Table 1. The parameters describing the flow rate between compartments $(Q)$ and the medium volumes in the liver and pancreas compartments $\left(V_{m, l i v e r}\right.$ and $V_{m, p a n c r e a s}$, respectively) were set to the actual MPS operating conditions during the experiment. The volume of HepaRG cells in the liver compartment ( $\left.V_{\text {HepaRG,spheroids }}\right)$ was estimated based on the number of liver spheroids in the co-culture (40) and the number of HepaRG cells per spheroid $(24,000)$, assuming an average hepatocyte volume 
of $3.4 \cdot 10^{-9} \mathrm{~cm}^{3}$ as reported in (Lodish, 2016). Similarly, the volume of pancreatic $\beta$ cells at the start of the co-culture $\left(V_{\beta, \text { islets }}(0)\right)$ was approximated from the number of pancreatic islets (10), assuming that the proportion of $\beta$ cells per islet is approximately $50 \%$, and that each islet was spherical with a diameter of $150 \mu \mathrm{m}$ (Insphero, 2016).

A subset of parameters characterizing insulin secretion and changes in volume of pancreatic $\beta$ cells $\left(\alpha_{G}, d_{0}, r_{1}\right.$ and $\left.r_{2}\right)$ were defined according to values reported in previous studies (Topp et al., 2000). The parameters that define normoglycemic concentrations in the co-culture $\left(G_{\text {normo }}\right)$ and $\tau_{\text {slow }}$ were approximated based on physiological considerations about the MPS system in the study, as previously described (Section 2.5). The remaining 12 parameters were estimated on a experiment-specific basis. This estimation was performed using nonlinear optimization, by finding parameter values that provided an acceptable agreement with the experimental data according to the following cost function:

$V(p)=\sum_{i} \sum_{t} \frac{\left(y_{i}(t)-\hat{y}_{i}(t, p)\right)^{2}}{S E M_{i}(t)^{2}}$

where $i$ is summed over the number of experimental time-series for the given experiment $y_{i}(t)$ and $\widehat{y}_{l}(t, p)$ represents the model simulations and $p$ the model parameters. SEM denotes the standard error of the mean and $t$ the measured time points in each time-series. Therefore, the value of the cost function $V(p)$ was calculated over all measured time points for all time-series considered in the optimization. We used a simulated annealing approach (Kirkpatrick et al., 1983) to find the set of acceptable parameters that provided good agreement with the data according to a statistical $\chi^{2}$ test (Cedersund and Roll, 2009; Cedersund, 2012). We chose a significance level of 0.05 , and the number of degrees of freedom was set to the number of data points in the experimental data.

\subsection{Software}

All computations were carried out in MATLAB R2018b (The Mathworks Inc., Natick, Massachusetts, USA) using IQM tools (IntiQuan GmbH, Basel, Switzerland) and the MATLAB Global Optimization toolbox. The freely available software WebPlotDigitizer 4.3 (https://automeris.io/WebPlotDigitizer) was used to extract the experimental data from the study by Dalla Man et al. (Man et al., 2007). Figures were prepared using BioRender (https://biorender.io/) and Illustrator CC 2019 (Adobe).

\subsection{Data correction}

The number of replicate platforms considered in the study was on average 5, varying between 4 and 10 across the different experiments. Due to this small sample size, we assume that the measured SEM is an underestimation of the uncertainty in the data and SEM values below 5\% of the corresponding mean are considered unrealistic. To correct for such possible underestimations in data uncertainty, we set the SEM of data points with a measured SEM below $5 \%$ of their mean to the largest measured SEM value across 
all points in the dataset. In experiments where all datapoints had a SEM value below 5\% of their mean, the SEM was changed to $10 \%$ of their mean instead. The resulting SEM values in each experiment are given as errors bars in the figures included in the main article and the Supplementary Information.

\section{Results}

\subsection{The integrated experimental-computational approach}

A flow chart of the steps involved in the experimental-computational approach is presented in Figure 3. First, we developed a computational model for the interplay between glucose and insulin in the liverpancreas MPS, describing fast (hours) glucose homeostasis and slow ( 2 weeks) changes in insulin sensitivity and $\beta$-cell dynamics (Figure 2). We limited the complexity of the model to represent only mechanisms needed to describe the experimental data in the study, thereby keeping the model's size small (12 free model parameters). A complete description of the model equations, as well as the code used for simulations, are provided in the Supplementary Material.

The next step was to calibrate the model on an experiment-specific basis. To perform this calibration, the parameters were estimated using the available data from the corresponding experiment. These data varied among the seven experiments (Supplementary Table 1), and comprised combinations of the following time-series measurements: 1) glucose and insulin concentrations during GTTs in co-cultures under two different glycemic regimes (hyper-, and/or normoglycemia) and 2) glucose concentrations during GTTs in single-liver cultures under hyperglycemia. The model development and calibration steps were executed in an iterative manner, allowing us to modify the model with each iteration until it was able to accurately describe the calibration data. The final model resulting from this iterative process is the one proposed in this paper. Last, we evaluated this model by testing its ability to predict data not considered during calibration in two of the seven experiments.

\subsection{Quantitative analysis of the mechanisms behind impaired glucose homeostasis over 15 days of co-culture}

We applied the computational model to quantitatively describe the physiological processes behind impaired glucose homeostasis in the liver-islet co-cultures. We calibrated the model using data from an experiment where both liver-islet and single-liver cultures were exposed to hyperglycemic conditions mimicking high plasma glucose in T2DM (experiment 2, Bauer et al., 2017). Figure 4 A-C shows a comparison between the model simulations and the experimental measurements used for calibration, which included time-series data of both glucose and insulin concentrations during GTTs in the cocultures (Fig. 4A and 4C respectively, red markers), and glucose concentration in the single-liver cultures (Fig.4B, blue markers). The estimated parameter values provided an acceptable agreement between the model simulations (Fig. 4 A-C, lines) and the data (Fig.4 A-C, markers), as determined by $\chi^{2}$ statistics (Supplementary Table S3). 
As shown in Fig. 4A, glucose levels in the liver-islet co-culture during the GTT initiated at day 1 (GTT d1-3) reached the normoglycemic range (3.9-7.8 $\mathrm{mM}$ (Bauer et al., 2017)) within eight hours, as opposed to the slower glucose consumption observed after 13 days of co-culture. In contrast, in single-liver cultures, glucose levels remained within hyperglycemia for the entire co-culture period (Fig.4B). These changes in glucose dynamics were accompanied by a decrease in insulin concentration levels over time, as seen in Fig. 4C. We used the modelling approach to infer variables that could mechanistically describe the physiological changes underlying these alterations in glucose regulation and $\beta$-cell function. According to the model, insulin sensitivity in the liver spheroids decreases progressively as they are exposed to hyperglycemic periods during the co-culture (Fig. 4E). This decline in insulin sensitivity leads to reduced glucose utilization by the liver spheroids, resulting in higher daily glucose levels over time (Fig. 4F). In turn, the model suggests an increase in the number of $\beta$-cells to compensate for the rise in glucose levels, and therefore $\beta$-cell volume increases (Fig. 4G). However, besides this adaptation in $\beta$-cell volume, the secretion capacity of the individual $\beta$-cells decays (Fig. $4 \mathrm{H}$ ). These combined effects result in a decline in circulating insulin levels over the 15-day co-culture period, in agreement with the experimental measurements (Fig. 4C).

We estimated parameters for each MPS experiment individually to fit the corresponding measurements of glucose and insulin, and achieved an acceptable agreement between the model simulations and the experimental data (Supplementary Table S2). A comparison between the model simulations and the experimental measurements for the seven experiments included in the study is shown in Supplementary Fig. S2. The estimated parameter values for each MPS experiment are listed in Supplementary Table S3.

\subsection{Investigating the effect of glycemic regimes on glucose metabolism}

To further investigate the effect of glycemic levels on liver spheroid-pancreatic islet cross-talk, we applied the computational model to leverage data from experiments under varying glycemic conditions. We performed in vitro experiments where co-cultures were exposed to both normo- and hyperglycemic glucose levels emulating healthy and T2DM conditions, respectively. By using the computational model, we sought to interpret the experimental results in relation to the changes in pancreatic $\beta$-cell function and impaired glucose tolerance.

We assessed how repeated exposure of the co-cultures to two different glycemic conditions (hyper- and normo-glycemia) impacted their response to a glucose load. To do so, the co-cultures were exposed to either hyper- or normoglycemic glucose levels (11 $\mathrm{mM}$ or $5.5 \mathrm{mM}$ glucose, respectively) at each medium exchange during the first 13 culture days. In the hyperglycemic co-cultures, we performed a GTT at day 1 (GTT d1-3, Fig. 5A,B) to evaluate glucose tolerance at the beginning of the co-culture. We then performed GTTs on day 13 (GTT d13-15, Fig. 5A,B) on both hyper- and normoglycemic cocultures, to establish a comparison with the response from the initial GTT for both glycemic regimes. 
The GTT initiated at day 1 was only performed in the co-cultures maintained under hyperglycemia, to avoid exposure of liver spheroids in normoglycemia to high glucose levels in the beginning of the coculture.

Analysis of the model simulations indicate that liver spheroids exposed to normoglycemic conditions over the co-culture period exhibited higher insulin sensitivities than those maintained under hyperglycemia (Fig. 5C). Thus, although insulin levels in hyperglycemic conditions during the GTT performed at day 13 were higher than those under normoglycemia (by 4 -fold $24 \mathrm{~h}$ after the start of the GTT, Fig. 5B), glucose levels were comparable for both glycemic regimes (Fig. 5A). These results are in line with our previous observations on the possible development of insulin resistance due to exposure to high glucose levels (Fig. 4). The observed differences in insulin secretion between hyper- and normoglycemia (Fig. 5B) could also be reproduced by the model. These differences could be explained by the bell-shaped relationship between average glucose levels in the co-culture and the net change of $\beta$-cell volume (Eq. 10). In the experiments under hyperglycemia, daily average glucose levels varied between 5.5 and $7.2 \mathrm{mM}$ over the the 15-day co-culture period (Fig. 5D). Thus, the model predicted a net increase in $\beta$-cell volume (Fig. 5E), as these values lie within the established range of glucose levels for increased rate of change of $\beta$-cell number (i.e. replication minus apoptosis), which is set to 5.55$13.87 \mathrm{mM}$ based on the study from Topp et al. (Topp et al., 2000). On the contrary, daily average glucose levels in co-cultures maintained under normoglycemia were within the range of 5.0-5.5 mM (Fig. 5D) postulated to lead to a decrease in $\beta$-cell volume (Fig. 5E) and the resulting decay in insulin secretion compared to hyperglycemia (Fig. 5B).

\subsection{Assessing the predictive capabilities of the computational model}

\subsubsection{Prediction of glucose and insulin responses under hypoglycemia}

To evaluate our computational model, we assessed whether it was able to predict data not employed during calibration (last step in Fig 3). To do so, we applied an experiment-specific model calibrated to both normo- and hyperglycemic conditions simultaneously (experiment 3, Fig. 5) to simulate hypoglycemia in silico. We then performed the corresponding in vitro MPS experiment, where the cocultures were exposed to hypoglycemic glucose levels $(2.8 \mathrm{mM})$ at each medium exchange during the first 13 culture days, followed by a GTT at day 13. To account for experimental uncertainties in the glucose dose administered to the system at the start of the GTT, we allowed the value of the glucose dose to vary within the measured range of SEM $( \pm 0.85 \mathrm{mM})$ when computing the model predictions.

The predictions of glucose and insulin responses during the GTT initiated at day 13 (GTT d13-15, Fig 6 B,D, shaded areas) were in good agreement with the experimental data (Fig 6 B,D, markers). 


\subsubsection{Prediction of long-term changes in glucose and insulin responses}

Next, we assessed the ability of the computational model to predict long-term changes in glucose and insulin responses in the liver-islet co-culture over time, and how these were influenced by the operating conditions in the MPS (i.e. the flow rate distribution on-chip and the medium volume in each culture compartment). To that end, in one experiment (experiment 3), we measured glucose and insulin concentrations periodically 48 hours after each media exchange between days 3 and 13 of the co-culture. In addition, to characterize the effect of the MPS operating conditions on the observed dynamics, we collected glucose and insulin samples from each culture compartment (liver and pancreas). The model was calibrated using glucose and insulin data acquired during GTTs initiated at day 1 and 13 (GTT d13 and GTT d13-15, Fig. 7 C,F and Fig. 4). In the experimental data used for calibration, the concentrations were measured by pooling samples of $15 \mu \mathrm{L}$ from each compartment (i.e. measuring the average concentration of the two compartments), as opposed to the compartment-wise measurements acquired for the evaluation dataset. The model predictions (Fig. 7 A,B,D,E, shaded areas) showed good agreement with the experimental measurements in terms of both temporal evolution of glucose and insulin dynamics along the co-culture, and the concentration levels in each culture compartment (Fig. 7 A,B,D,E, markers). More specifically, the computational model captured the greater insulin concentration in the pancreas compartment compared to that in the liver compartment.

\subsection{Translation to in vivo responses in humans}

Following evaluation, we investigated whether a model-based scaling strategy could translate the MPS results from in vitro to in vivo. We established an upscaling approach that involves extrapolation of the following model parameters: volumes of the organoids, flow rate and volume of co-culture medium in the compartments. To upscale the volume of the organoids to human proportions, we multiplied the total volume of both HepaRG cells ( $\left.V_{\text {HepaRG,spheroids }}\right)$ and pancreatic $\beta$ cells $\left(V_{\beta, \text { islets }}(0)\right)$ by the 100,000 factor applied in the miniaturization to MPS. The total volume of co-culture medium was scaled to $3 \mathrm{~L}$, under the assumption that the blood volume in humans is approximately 5.1 L (Wagner et al., 2013) with a plasma proportion of $58 \%$ (Feher, 2012). This volume was distributed equally between both tissue compartments, resulting in a medium volume of $1.5 \mathrm{~L}$ in each compartment. The flow rate $(Q)$ was then set to achieve a media turnover time of $5 \mathrm{~min}$, as observed in humans (Davies and Morris, 1993).

As a proof-of-concept demonstration, we tested the scaling strategy in one of the experiments (experiment 1). We first calibrated the original model using data of glucose and insulin during GTTs initiated at day 1 (GTT d1-3) in both liver-islet co-cultures and single-liver cultures (Supplementary Fig. S2 A-C, markers). The parameters describing the operating conditions in the system were then modified according to the proposed upscaling approach. Additionally, to account for glucose consuming organs other than the liver (i.e. muscle, adipose tissue, brain and kidneys), we increased the glucose uptake rate of the liver. More specifically, we multiplied both the insulin-independent glucose disposal rate $\left(E_{G 0}\right)$ 
and insulin sensitivity $\left(S_{I}\right)$ by a factor of 2.22 , assuming that the liver is responsible for approximately $45 \%$ of the total postprandial glucose uptake in humans (Gerich, 2000; Herrgårdh et al., 2021). Similarly, the insulin clearance of the liver $\left(C L_{I, h e p}\right)$ was doubled under the assumption that the liver stands for approximately $50 \%$ of the total insulin clearance (Becker, 2001).

After these parameter changes, the temporal dynamics of the glucose and insulin responses predicted in the model were significantly faster than in the MPS and in agreement with those found in humans (Man et al., 2007) (Supplementary Fig. S3 C,F). However, the glucose uptake predicted by the model (Supplementary Fig. S3 C, shaded area) was larger than the in vivo measurement (Supplementary Fig. S3 C, markers). Furthermore, the predicted insulin concentrations (Supplementary Fig. S3 F, shaded area) were one order of magnitude higher than the ones measured in vivo (Supplementary Fig. S3 F, markers). The peak insulin concentration in the model predictions ranged between 1.8 and $2.0 \mathrm{nM}$, while the corresponding in vivo value was $0.34 \mathrm{nM}$. Because insulin concentrations measured in the MPS were also one order of magnitude higher than those reported in human studies (Bergman et al., 1981; Man et al., 2007; Alskär et al., 2017), we hypothesize that the pancreatic islets in the co-culture may have enhanced insulin secretion compared to the human in situ case. This can possibly be due to the longterm exposure of the pancreatic islets to a hyperglycemic medium over the co-culture time, leading to overstimulation of insulin release. To account for this potential effect, we reduced the maximal insulin secretion rate of the $\beta$ cells $\left(\sigma_{\max }\right)$, adjusting its value to achieve good visual agreement with the insulin concentration measured in vivo. The changes in model parameters for translation from the liver-islet MPS to human for a parameter set providing acceptable agreement with the experimental data are listed in Table 2. The resulting glucose and insulin responses predicted by the computational model (Fig. 8 C,F, shaded areas) agree well with the measured ones in humans (Fig. 8 C,F, markers), even though the predicted glucose concentration decreases to values below normoglycemia as endogenous glucose production from the liver spheroids cells is neglected in our model.

\section{Discussion}

This study demonstrated the potential of applying computational modelling in combination with MPS to augment in vitro investigations of glucose metabolism and allow translation to humans. We constructed a computational model of glucose homeostasis in a previously developed liver-pancreas MPS (Bauer et al., 2017). After calibration, the model was able to replicate glucose and insulin responses under both healthy glucose levels and high plasma glucose mimicking T2DM (Fig. 5). To demonstrate the predictive power of the model, we evaluated it on measurements not considered for calibration. The model could correctly predict the response of the MPS to a hypoglycemic regime (Fig. 6), and the longterm dynamics of glucose and insulin over 15 days of co-culture (Fig. 7). Last, we have shown that the model is able to translate in vitro glucose and insulin responses in the MPS to humans, showing good agreement with reported data on meal responses from healthy subjects (Man et al., 2007) (Fig. 8). 
The mechanistic, computational model presented in this study aims at describing the physiology in the MPS, encapsulating mechanisms underlying glucose regulation and disease progression in T2DM (insulin resistance and $\beta$-cell adaptation). This is an advancement from the models that have typically been combined with MPS, which mainly apply PK (Prot et al., 2014; Kimura et al., 2015; Edington et al., 2017; Lee, Ha, et al., 2017) and PKPD (Sung et al., 2010; Lee, Kim, et al., 2017; McAleer et al., 2019; Novak et al., 2020) approaches to describe the pharmacological effect of drugs in the system. Only a few studies have combined MPS with QSP models that include more mechanistic details (Stokes et al., 2018; Maass et al., 2019; Yu et al., 2015). Lee et al. (Lee et al., 2019) developed a computational model of glucose homeostasis in a pancreas-muscle MPS using in vitro measurements from individual cell cultures, and added a liver compartment in silico as a step towards translation to in vivo responses. This study, however, did not include experimental measurements characterizing the cross-talk between these organs. Since glucose homeostasis relies on a feedback loop involving storage and release of glucose by the liver in response to glucose-regulated insulin secretion from the pancreas, it is crucial to examine data from interconnected co-cultures that reflect this organ interplay. Moreover, the computational model in Lee et al. (Lee et al., 2019) was designed to simulate a 3-hour response to a meal, and, in contrast to our model, did not include a description of disease progression.

Several computational approaches have also been developed to describe glucose homeostasis and different aspects of T2DM in animal models. These could potentially be used in combination with preclinical animal studies, to help in data interpretation and extrapolating the results to humans. Alskär et al. (Alskär et al., 2017) demonstrated that an allometrically scaled model of human glucose homeostasis (Silber et al., 2007) could reproduce glucose and insulin responses in several preclinical animal species. However, this model only describes short-term regulation of glucose homeostasis during GTT and cannot simulate the long-term pathophysiology of T2DM. In contrast, other animal-based computational models have focused on long-term changes in weight (Guo and Hall, 2011; Gennemark et al., 2013), but they do not establish a link with pathophysiological defects implicated in T2DM such as insulin resistance.

The computational model presented in our study could simultaneously describe glucose and insulin responses in the MPS under both normo- and hyperglycemic conditions representative of T2DM (Fig. $5 \mathrm{~A}, \mathrm{~B})$. The fact that the model can reproduce a range of behaviors consistent with experimental observations only through estimation of the model parameters suggests the generality of the model structure, that is, the robustness of the mathematical equations. However, to further build confidence in the computational model, it is crucial to evaluate its predictive capability against experimental data not considered during calibration (Carusi et al., 2012; Pathmanathan and Gray, 2018). Considering this, we first calibrated the model using MPS measurements from normo- and hyper-glycemic conditions, and subsequently evaluated the model predictions under a different glycemic regime (hypoglycemia) using 
data from an independent MPS experiment. We showed that the model was able to predict glucose and insulin responses under this new glycemic regime, although it was not calibrated for this purpose (Fig. $6 \mathrm{C}, \mathrm{D})$. In addition, we demonstrated the ability of the model to predict glucose and insulin concentrations at times not included in our experimental sampling protocol, and in both culture compartments (Fig. 6 A,B,D,E).

Our approach also offers mechanistic insight through simulation of key physiological variables that are not measured in the experiments, such as insulin resistance and the total volume of $\beta$ cells in the coculture (Figs. 4,5). Our model predicted an increase in total $\beta$-cell volume after exposure to hyperglycemic glucose levels over 15 days of co-culture, while this volume was predicted to decrease when the pancreatic islets were exposed to normoglycemic levels (Fig. 5E). These predictions agree with the previously established hypothesis that small deviations from normoglycemia cause $\beta$-cell volume increase as a feedback mechanism to reestablish glucose homeostasis (Topp et al., 2000), which stems from previous studies reporting a nonlinear variation of both $\beta$-cell proliferation and apoptosis rates in vitro (Swenne, 1982; Hügl et al., 1998). GSIS analysis from our co-culture experiments revealed increased insulin secretion in pancreatic islets co-cultured under hyperglycemic conditions $(11 \mathrm{mM})$ as compared to those in either normo- or hypoglycemic co-cultures (5.5 and $2.8 \mathrm{mM}$, respectively) (Supplementary Fig. S1). This might indicate that the total volume of $\beta$ cells in the end of the co-culture is larger when they have been exposed to hyperglycemic concentrations. However, GSIS measurements do not only reflect the volume of $\beta$-cells but also their individual secretion capacity. To increase confidence in this model prediction, future studies should be carried out to evaluate beta cell volumes in the liver-islet co-culture under different glycemic levels, for instance focusing on beta cell proliferation.

The complexity of the computational model was chosen based on the intended level of detail and the available experimental measurements. With this in mind, we only modelled the physiological mechanisms needed to simulate the data in the study. Here, the only measurements available to characterize the contribution of the liver spheroids to glucose metabolism were glucose concentrations in the co-culture medium over time. Therefore, we established a relatively simple model of hepatic glucose metabolism that only captured net glucose fluxes between the liver spheroids and the co-culture medium, but did not describe any intracellular fluxes. Additional experiments using isotope labeling tracing methods (Landau et al., 1995; Neese et al., 1995; Grankvist et al., 2018) could be done to characterize metabolic pathways in the liver spheroids, including both glucose producing (e.g. glycolysis and glyconeogenesis) and glucose utilizing (e.g. gluconeogenesis, glycogenolysis) pathways. We may then expand our current computational framework by incorporating more detailed models of hepatic glucose metabolism (König et al., 2012; Ashworth et al., 2016) for further elucidation of mechanisms behind T2DM and refined in silico predictions. 
Because of the relatively small number of MPS experiments included in our study, we calibrated the computational model using data exclusively from a given individual experiment. By acquiring data from a larger set of MPS experiments, we could expand our framework with a non-linear mixed-effects (NLME) modelling approach (Karlsson et al., 2015). Using this approach, we could estimate the model parameters using data from all the MPS experiments simultaneously, thereby sharing information among them. This would in turn allow us to obtain additional insight on interindividual variability within the experiments, and more robust parameter estimation when the available data from one experiment alone may be insufficient.

The most widespread models to study glucose metabolism under both healthy and T2DM conditions are in vivo animal models in rodents. These are comparators for the novel, integrated experimental (MPS)computational approach presented here. Translation between these animal models and humans is often unsuccessful, partly due to species-specific glucose regulation mechanisms ranging from cell to organ level (Chandrasekera and Pippin, 2014). To overcome these limitations, the liver-pancreas MPS in this study incorporates human cells at organ emulation levels (e.g. 3D tissue environment), mimicking their human counterpart architecture and function. While our system could potentially be more predictive of in vivo, human outcome, its ability to replicate human physiology is still limited. For instance, with our current experimental setting, kinetics of the in vitro glucose response was considerably slower than in vivo. In our liver-pancreas MPS, glucose levels reached normoglycemia approximately 48 hours after the start of a GTT, as opposed to 1 to 2 hours in vivo (Bergman et al., 1981; Man et al., 2007; Fujii et al., 2019). This could be due to several factors including design aspects, properties of the co-culture medium and the organoids, operating conditions (e.g. flow rate) and other glucose consuming organs and signaling mechanisms (e.g. incretins). For example, the media-to-tissue ratio in our system was in the order of 100:1, whereas the physiological extracellular fluid to tissue ratio is 1:4 (Wagner et al., 2013). This could potentially cause the slower GTT response in our system, since the proportion between total mass of glucose and glucose-consuming liver cells is larger than in the in vivo case. The synergistic experimental-computational approach allowed us to gain insight into the impact of these experimental in vitro factors in the system and ultimately bridge the in vitro-in vivo gap by compensating for them in silico. We argue that to maximize the ability of this approach to exploit the in vitro MPS investigations, it should be applied in an iterative fashion that involves two steps: data interpretation/translation and model-guided design.

In the data interpretation/translation step, we calibrated the computational model using glucose and insulin responses measured during GTTs in the liver-pancreas MPS and performed model-based extrapolation (i.e. scaling) of in vitro experimental aspects (co-culture and organoid volumes, flow rates and incorporation of missing organs) to translate to humans. After this extrapolation, both insulin levels (Supplementary Fig. S3 C, shaded areas) and glucose uptake (Supplementary Fig. S3 F, shaded areas) 
were higher than those observed during meal responses in humans (black markers, from Man et al., 2007). These discrepancies indicate that our current MPS set-up may have design limitations that cannot be compensated for using a purely scaling approach, thereby pinpointing possible biological imperfections related to both the organoids and co-culture conditions. One potential explanation concerning biological imperfections is that the increased insulin levels in the MPS (Fig. S3F) are due to enhanced insulin secretion of the pancreatic islets compared to in vivo. Other possible explanations would be the lack of sinusoidal structures in the liver model (Meier, 2005), and the lack of renal insulin clearance mechanisms (Meier, 2005). As a proof-of-concept investigation, we tested the first potential explanation using the computational model by decreasing the insulin secretion of the pancreatic islets in silico. The simulated glucose and insulin responses agreed well with the experimental data from humans (Fig. 8 C,F), suggesting that a decreased insulin secretion capacity of the pancreatic islets in the MPS in combination with the model-based scaling approach would yield human-like responses. However, this result does not imply that the remaining explanations should be rejected, and they can be tested in a similar manner using the computational model. These mechanistic insights could be used in a future model-guided design step, to enhance the physiological relevance of our in vitro MPS through, for instance, increased insulin clearance by the liver organoid or decreased insulin secretion by the pancreatic islets. This updated MPS set-up would then generate experimental data that supports the development of a respective, extended computational model for prediction in the subsequent data interpretation/translation step.

A recent MPS report to advance patient's benefit and animal welfare has identified four elements to make preclinical drug evaluation predictive to human exposure (Marx et al., 2020). These elements are: i) academic invention and MPS-model development, ii) tool creation and MPS-model qualification by supplier industries, iii) qualification of a fit-for-purpose assay and its adoption for candidate testing by pharmaceutical industries, and iv) regulatory acceptance of the predictive results of validated assays for a drug candidate for a specific context of use. Here, we propose to support these MPS-based developments with computational modelling. Our results demonstrate the synergies between MPS and computational models, which we believe would accelerate the drug development cycle. Both in academic science and pharmaceutical decision making, fit-for-purpose experimental-computational models hold the potential to reduce the use of animal models currently used for the same purpose. A purely experimental in vitro approach to this goal is further away, since recreating human-like responses in vitro poses major challenges related to constraints in design and experimental conditions. For example, the differences in media-to-tissue ratio that lead to the slow time dynamics in GTT responses in our MPS system are difficult to address experimentally, because a reduction in the volume of coculture medium would result in sampling volumes that are insufficient for analysis. With our integrated experimental-computational approach these limitations can be overcome. Our vision is that once the effect of drugs are well-characterized in vitro in MPS recapitulating the physiology of different organs, 
computational modelling can be used to create in silico representations of individual organs that can then be combined in a multi-organ computational model as a step towards extrapolation to humans.

In recent years, evidence generated by computational models has drawn increasing attention from regulatory agencies. The United States Food and Drug Administration (FDA) has published guidance on assessing the credibility of computational models for regulatory submissions (summarized in (Viceconti et al., 2021)). The assessment should be performed within a specific context of use in which the model is intended to be applied, and requires validation of the model output against comparator data (e.g experimental measurements) not considered during calibration. Two metrics should be considered in this validation: the agreement between the model predictions and the comparator data, and the uncertainty of these predictions. In this paper, we have created a computational model that can describe experimental data not used for calibration purposes with well-defined uncertainty boundaries (Figs. 6,7). Once the corresponding human-based predictions (as exemplified in Fig. 8) have been validated within a context of use relevant to a clinical trial, the integrated experimental-modelling approach might eventually be submitted for regulatory qualification. We envision that, when fully incorporated into the early stages of drug development, this approach could reduce animal experiments and significantly decrease phase 1 and phase 2 clinical trial failures due to its relatively low cost and ability to generate human-relevant predictions.

\section{Data availability}

All data supporting the results in this study are available in the main article and the Supplementary Information. Codes to reproduce all the figures are available at https://github.com/belencasasgarcia/Liver-islet-MPS.git

\section{Acknowledgements}

We acknowledge funding from the Swedish research council (2018-05418, 2018-03319), CENIIT (15.09), the Swedish foundation for strategic research (ITM17-0245), SciLifeLab/KAW national COVID-19 research program project grant (2020.0182), H2020 (PRECISE4Q, 777107), The Swedish Fund for Research without Animal Experiments, and ELLIIT.

\section{Conflicts of interest}

LV, KK, CWH, LM, TBA, PG are employees of AstraZeneca and hold stock/stock options 


\section{References}

Alskär, O., Karlsson, M. O. and Kjellsson, M. C. (2017). Model-Based Interspecies Scaling of Glucose Homeostasis. CPT pharmacometrics Syst Pharmacol 6, 778-786. https://doi.org/10.1002/psp4.12247.

Ashworth, W. B., Davies, N. A. and Bogle, I. D. L. (2016). A Computational Model of Hepatic Energy Metabolism: Understanding Zonated Damage and Steatosis in NAFLD. PLoS Comput Biol 12, 1-30. https://doi.org/10.1371/journal.pcbi.1005105.

Balducci, S. (2014). Regulation of hepatic glucose production and the role of gluconeogenesis in humans: is the rate of gluconeogenesis constant? Diabetes Metab Res Rev 32, 13-23. https://doi.org/10.1002/dmrr.

Banaeiyan, A. A., Theobald, J., Paukštyte, J. et al. (2017). Design and fabrication of a scalable liverlobule-on-a-chip microphysiological platform. Biofabrication 9, 15014. https://doi.org/10.1088/1758-5090/9/1/015014.

Bauer, S., Wennberg Huldt, C., Kanebratt, K. P. et al. (2017). Functional coupling of human pancreatic islets and liver spheroids on-a-chip: Towards a novel human ex vivo type 2 diabetes model. Sci Rep 7, 14620. https://doi.org/10.1038/s41598-017-14815-w.

Becker, K. L. (2001). Principles and practice of endocrinology and metabolism. Third edit. Lippincott Williams \& Wilkins. Available at: https://login.e.bibl.liu.se/login?url=https://search.ebscohost.com/login.aspx?direct=true \&db=cat0 0115a\&AN=lkp.764565\&site $=$ eds-live \&scope $=$ site.

Bergman, R. N. (1989). Toward physiological understanding of glucose tolerance. Minimal-model approach. Diabetes 38, 1512-1527. https://doi.org/10.2337/diab.38.12.1512.

Bergman, R. N., Ider, Y. Z., Bowden, C. R. et al. (1979). Quantitative estimation of insulin sensitivity. Am J Physiol Metab 236, E667. https://doi.org/10.1152/ajpendo.1979.236.6.E667.

Bergman, R. N., Phillips, L. S. and Cobelli, C. (1981). Physiologic evaluation of factors controlling glucose tolerance in man: measurement of insulin sensitivity and beta-cell glucose sensitivity from the response to intravenous glucose. J Clin Invest 68, 1456-1467. https://doi.org/10.1172/JCI110398.

Bernard, C., Thibault, C., Berthault, M. F. et al. (1998). Pancreatic $\beta$-cell regeneration after 48-h glucose infusion in mildly diabetic rats is not correlated with functional improvement. Diabetes 47, 1058-1065. https://doi.org/10.2337/diabetes.47.7.1058.

Bonner-Weir, S., Deery, D., Leahy, J. L. et al. (1989). Compensatory Growth of Pancreatic $\beta$-Cells in Adult Rats After Short-Term Glucose Infusion. Diabetes 38, 49. https://doi.org/10.2337/diab.38.1.49.

Calcutt, N. A., Cooper, M. E., Kern, T. S. et al. (2009). Therapies for hyperglycaemia-induced diabetic complications: From animal models to clinical trials. Nat Rev Drug Discov 8, 417-429. https://doi.org/10.1038/nrd2476.

Carusi, A., Burrage, K. and Rodríguez, B. (2012). Bridging experiments, models and simulations: an integrative approach to validation in computational cardiac electrophysiology. Am J Physiol Circ Physiol 303, H144-H155. https://doi.org/10.1152/ajpheart.01151.2011.

Cedersund, G. (2012). Conclusions via unique predictions obtained despite unidentifiability - new definitions and a general method. FEBS J 279, 3513-3527. https://doi.org/10.1111/j.17424658.2012.08725.x.

Cedersund, G. and Roll, J. (2009). Systems biology: model based evaluation and comparison of potential explanations for given biological data. FEBS J 276, 903-922. https://doi.org/10.1111/j.1742-4658.2008.06845.x.

Chandrasekera, P. C. and Pippin, J. J. (2014). Of rodents and men: Species-specific glucose regulation and type 2 diabetes research. ALTEX 31, 157-176. https://doi.org/10.14573/altex.1309231.

Chick, W. L. (1973). Beta Cell Replication in Rat Pancreatic Monolayer Cultures: Effects of Glucose, Tolbutamide, Glucocorticoid, Growth Hormone and Glucagon. Diabetes 22, 687 LP - 693. https://doi.org/10.2337/diab.22.9.687.

Cirit, M. and Stokes, C. L. (2018). Maximizing the impact of microphysiological systems with in vitro-in vivo translation. Lab Chip 18, 1831-1837. https://doi.org/10.1039/C8LC00039E.

Davidson, M. D., Ballinger, K. R. and Khetani, S. R. (2016). Long-term exposure to abnormal glucose 
levels alters drug metabolism pathways and insulin sensitivity in primary human hepatocytes. Sci Rep 6, 28178. https://doi.org/10.1038/srep28178.

Davies, B. and Morris, T. (1993). Physiological Parameters in Laboratory Animals and Humans. Pharm Res 10, 1093-1095. https://doi.org/10.1023/A:1018943613122.

Du, Y., Li, N., Yang, H. et al. (2017). Mimicking liver sinusoidal structures and functions using a 3Dconfigured microfluidic chip. Lab Chip 17, 782-794. https://doi.org/10.1039/C6LC01374K.

Edington, C. D., Cirit, M., Chen, W. L. K. et al. (2017). Integration of systems biology with organson-chips to humanize therapeutic development. In Proc.SPIE. Available at: https://doi.org/10.1117/12.2256078.

Efanova, I. B., Zaitsev, S. V., Zhivotovsky, B. et al. (1998). Glucose and tolbutamide induce apoptosis in pancreatic $\beta$-cells: A process dependent on intracellular Ca2+ concentration. $J$ Biol Chem 273, 33501-33507. https://doi.org/10.1074/jbc.273.50.33501.

Esch, E. W., Bahinski, A. and Huh, D. (2015). Organs-on-chips at the frontiers of drug discovery. Nat Rev Drug Discov 14, 248-260. https://doi.org/10.1038/nrd4539.

Feher, J. (2012). 5.13 - Regulation of Arterial Pressure. In J. B. T.-Q. H. P. Feher (ed.), (538-548). Boston: Academic Press. https://doi.org/https://doi.org/10.1016/B978-0-12-382163-8.00058-X.

Fujii, M., Murakami, Y., Karasawa, Y. et al. (2019). Logical design of oral glucose ingestion pattern minimizing blood glucose in humans. npj Syst Biol Appl 5, 31. https://doi.org/10.1038/s41540019-0108-1.

Gennemark, P., Jansson-Löfmark, R., Hyberg, G. et al. (2013). A modeling approach for compounds affecting body composition. $J$ Pharmacokinet Pharmacodyn 40, 651-667. https://doi.org/10.1007/s10928-013-9337-x.

Gerich, J. E. (2000). Physiology of glucose homeostasis. Diabetes, Obes Metab 2, 345-350. https://doi.org/10.1046/j.1463-1326.2000.00085.x.

van der Graaf, P. H. and Benson, N. (2011). Systems Pharmacology: Bridging Systems Biology and Pharmacokinetics-Pharmacodynamics (PKPD) in Drug Discovery and Development. Pharm Res 28, 1460-1464. https://doi.org/10.1007/s11095-011-0467-9.

Grankvist, N., Watrous, J. D., Lagerborg, K. A. et al. (2018). Profiling the Metabolism of Human Cells by Deep 13C Labeling. Cell Chem Biol 25, 1419-1427.e4. https://doi.org/10.1016/j.chembiol.2018.09.004.

Guo, J. and Hall, K. D. (2011). Predicting changes of body weight, body fat, energy expenditure and metabolic fuel selection in C57BL/6 mice. PLoS One 6, 1-9. https://doi.org/10.1371/journal.pone.0015961.

Ha, J., Sherman, A. S. and Satin, L. S. (2015). A Mathematical Model of the Pathogenesis, Prevention, and Reversal of Type 2 Diabetes. Endocrinology 157, 624-635. https://doi.org/10.1210/en.20151564.

Herrgårdh, T., Li, H., Nyman, E. et al. (2021). An Updated Organ-Based Multi-Level Model for Glucose Homeostasis: Organ Distributions, Timing, and Impact of Blood Flow. Front Physiol 12, 1-16. https://doi.org/10.3389/fphys.2021.619254.

Hoorens, A., Van de Casteele, M., Klöppel, G. et al. (1996). Glucose promotes survival of rat pancreatic beta cells by activating synthesis of proteins which suppress a constitutive apoptotic program. J Clin Invest 98, 1568-1574. https://doi.org/10.1172/JCI118950.

Hügl, S. R., White, M. F. and Rhodes, C. J. (1998). Insulin-like growth factor I (IGF-I)-stimulated pancreatic $\beta$-cell growth is glucose-dependent synergistic activation of insulin receptor substratemediated signal transduction pathways by glucose and IGF-I in INS- 1 cells. J Biol Chem 273, 17771-17779. https://doi.org/10.1074/jbc.273.28.17771.

Insphero (2016). 3D InSight ${ }^{\mathrm{TM}}$ Islet Microtissues : An advanced in vitro islet model for diabetes research. 2016, 1-11. Available at: https://insphero.com/wpcontent/uploads/2017/08/3D_InSight_Islets_white_paper.pdf [Accessed May 28, 2021].

Jullesson, D., Johansson, R., Rajan, M. R. et al. (2015). Dominant negative inhibition data should be analyzed using mathematical modeling - re-interpreting data from insulin signaling. FEBS J 282, 788-802. https://doi.org/https://doi.org/10.1111/febs.13182.

Karlsson, M., Janzén, D. L. I., Durrieu, L. et al. (2015). Nonlinear mixed-effects modelling for single cell estimation: when, why, and how to use it. BMC Syst Biol 9, 52. https://doi.org/10.1186/s12918-015-0203-x. 
Kimura, H., Ikeda, T., Nakayama, H. et al. (2015). An On-Chip Small Intestine-Liver Model for Pharmacokinetic Studies. J Lab Autom 20, 265-273. https://doi.org/10.1177/2211068214557812.

Kirkpatrick, S., Gelatt, C. D. and Vecchi, M. P. (1983). Optimization by Simulated Annealing. Science (80- ) 220, 671 LP - 680. https://doi.org/10.1126/science.220.4598.671.

König, M., Bulik, S. and Holzhütter, H. G. (2012). Quantifying the contribution of the liver to glucose homeostasis: A detailed kinetic model of human hepatic glucose metabolism. PLoS Comput Biol 8. https://doi.org/10.1371/journal.pcbi.1002577.

Landau, B. R., Wahren, J., Chandramouli, V. et al. (1995). Use of $2 \mathrm{H} 2 \mathrm{O}$ for estimating rates of gluconeogenesis: Application to the fasted state. J Clin Invest 95, 172-178. https://doi.org/10.1172/JCI117635.

Lee, D. W., Ha, S. K., Choi, I. et al. (2017). 3D gut-liver chip with a PK model for prediction of firstpass metabolism. Biomed Microdevices 19, 100. https://doi.org/10.1007/s10544-017-0242-8.

Lee, D. W., Lee, S. H., Choi, N. et al. (2019). Construction of pancreas-muscle-liver microphysiological system (MPS) for reproducing glucose metabolism. Biotechnol Bioeng 0. https://doi.org/10.1002/bit.27151.

Lee, H., Kim, D. S., Ha, S. K. et al. (2017). A pumpless multi-organ-on-a-chip (MOC) combined with a pharmacokinetic-pharmacodynamic (PK-PD) model. Biotechnol Bioeng 114, 432-443. https://doi.org/https://doi.org/10.1002/bit.26087.

Lee, P. J., Hung, P. J. and Lee, L. P. (2007). An artificial liver sinusoid with a microfluidic endothelial-like barrier for primary hepatocyte culture. Biotechnol Bioeng 97, 1340-1346. https://doi.org/10.1002/bit.21360.

Lodish, U. H. (2016). Molecular Cell Biology. W.H. Freeman.

Maass, C., Sorensen, N. B., Himmelfarb, J. et al. (2019). Translational Assessment of Drug-Induced Proximal Tubule Injury Using a Kidney Microphysiological System. CPT Pharmacometrics Syst Pharmacol 8, 316-325. https://doi.org/10.1002/psp4.12400.

Malaisse, W., Malaisse-Lagae, F. and Wright, P. H. (1967). A New Method for the Measurement in Vitro of Pancreatic Insulin Secretion. Endocrinology 80, 99-108. https://doi.org/10.1210/endo80-1-99.

Man, C. D., Rizza, R. A. and Cobelli, C. (2007). Meal Simulation Model of the Glucose-Insulin System. IEEE Trans Biomed Eng 54, 1740-1749. https://doi.org/10.1109/TBME.2007.893506.

Marx, U., Akabane, T., Andersson, T. B. et al. (2020). Biology-inspired microphysiological systems to advance patient benefit and animal welfare in drug development. ALTEX 37, 364-394. https://doi.org/10.14573/altex.2001241.

Marx, U., Andersson, T. B., Bahinski, A. et al. (2016). Biology-inspired microphysiological system approaches to solve the prediction dilemma of substance testing. ALTEX 33, 272-321. https://doi.org/10.145/3/aitex.1603161.

Materne, E. M., Tonevitsky, A. G. and Marx, U. (2013). Chip-based liver equivalents for toxicity testing-organotypicalness versus cost-efficient high throughput. Lab Chip 13, 3481-3495. https://doi.org/10.1039/c3lc50240f.

McAleer, C. W., Pointon, A., Long, C. J. et al. (2019). On the potential of in vitro organ-chip models to define temporal pharmacokinetic-pharmacodynamic relationships. Sci Rep 9, 1-14. https://doi.org/10.1038/s41598-019-45656-4.

Meier, J. J. (2005). Pulsatile insulin secretion. Novartis Found Symp 227, 190-205. https://doi.org/10.1002/0470870796.ch11.

Narang, A. S. and Mahato, R. I. (2006). Biological and Biomaterial Approaches for Improved Islet Transplantation. Pharmacol Rev 58, 194 LP - 243. https://doi.org/10.1124/pr.58.2.6.

Neese, R. A., Schwarz, J. M., Faix, D. et al. (1995). Gluconeogenesis and intrahepatic triose phosphate flux in response to fasting or substrate loads. Application of the mass isotopomer distribution analysis technique with testing of assumptions and potential problems. $J$ Biol Chem 270, 1445214463. https://doi.org/10.1074/jbc.270.24.14452.

Novak, R., Ingram, M., Marquez, S. et al. (2020). Robotic fluidic coupling and interrogation of multiple vascularized organ chips. Nat Biomed Eng 4, 407-420. https://doi.org/10.1038/s41551019-0497-x.

Nyman, E., Lindgren, I., Lövfors, W. et al. (2015). Mathematical modeling improves EC50 estimations from classical dose-response curves. FEBS J 282, 951-962. 
https://doi.org/https://doi.org/10.1111/febs.13194.

Olson, H., Betton, G., Robinson, D. et al. (2000). Concordance of the toxicity of pharmaceuticals in humans and in animals. Regul Toxicol Pharmacol 32, 56-67. https://doi.org/10.1006/rtph.2000.1399.

Ortega-Prieto, A. M., Skelton, J. K., Wai, S. N. et al. (2018). 3D microfluidic liver cultures as a physiological preclinical tool for hepatitis B virus infection. Nat Commun 9. https://doi.org/10.1038/s41467-018-02969-8.

Paris, M., Bernard-Kargar, C., Berthault, M. F. et al. (2003). Specific and combined effects of insulin and glucose on functional pancreatic $\beta$-cell mass in vivo in adult rats. Endocrinology 144, 2717 2727. https://doi.org/10.1210/en.2002-221112.

Pathmanathan, P. and Gray, R. A. (2018). Validation and trustworthiness of multiscale models of cardiac electrophysiology. Front Physiol 9, 1-19. https://doi.org/10.3389/fphys.2018.00106.

Petersen, M. C. and Shulman, G. I. (2018). Mechanisms of Insulin Action and Insulin Resistance. Physiol Rev 98, 2133-2223. https://doi.org/10.1152/physrev.00063.2017.

Prot, J. M., Maciel, L., Bricks, T. et al. (2014). First pass intestinal and liver metabolism of paracetamol in a microfluidic platform coupled with a mathematical modeling as a means of evaluating ADME processes in humans. Biotechnol Bioeng 111, 2027-2040. https://doi.org/10.1002/bit.25232.

Schimek, K., Busek, M., Brincker, S. et al. (2013). Integrating biological vasculature into a multiorgan-chip microsystem. Lab Chip 13, 3588-3598. https://doi.org/10.1039/c3lc50217a.

Silber, H. E., Jauslin, P. M., Frey, N. et al. (2007). An Integrated Model for Glucose and Insulin Regulation in Healthy Volunteers and Type 2 Diabetic Patients Following Intravenous Glucose Provocations. J Clin Pharmacol 47, 1159-1171. https://doi.org/10.1177/0091270007304457.

Stokes, C. L., Cirit, M., LaBarge, M. E. et al. (2018). Establishing quasi-steady state operations of microphysiological systems (MPS) using tissue-specific metabolic dependencies. Sci Rep 8, 113. https://doi.org/10.1038/s41598-018-25971-y.

Stokes, C. L., Cirit, M. and Lauffenburger, D. A. (2015). Physiome-on-a-Chip: The Challenge of "scaling" in Design, Operation, and Translation of Microphysiological Systems. CPT Pharmacometrics Syst Pharmacol 4, 559-562. https://doi.org/10.1002/psp4.12042.

Stumvoll, M., Goldstein, B. J. and van Haeften, T. W. (2005). Type 2 diabetes: principles of pathogenesis and therapy. Lancet 365, 1333-1346. https://doi.org/https://doi.org/10.1016/S01406736(05)61032-X.

Sung, J. H., Kam, C. and Shuler, M. L. (2010). A microfluidic device for a pharmacokineticpharmacodynamic (PK-PD) model on a chip. Lab Chip 10, 446-455. https://doi.org/10.1039/b917763a.

Sung, J. H., Wang, Y. and Shuler, M. L. (2019). Strategies for using mathematical modeling approaches to design and interpret multi-organ microphysiological systems (MPS). APL Bioeng 3, 21501. https://doi.org/10.1063/1.5097675.

Swenne, I. (1982). The role of glucose on the in vitro regulation of cell cycle kinetics and proliferation of fetal pancreatic B-cells. Diabetes 31, 754-760. https://doi.org/10.2337/diab.31.9.754.

Topp, B. G., Atkinson, L. L. and Finegood, D. T. (2007). Dynamics of insulin sensitivity, $\beta$-cell function, and $\beta$-cell mass during the development of diabetes in fa/fa rats. Am J Physiol Metab 293, E1730-E1735. https://doi.org/10.1152/ajpendo.00572.2007.

Topp, B., Promislow, K., Devries, G. et al. (2000). A model of $\beta$-cell mass, insulin, and glucose kinetics: Pathways to diabetes. J Theor Biol 206, 605-619. https://doi.org/10.1006/jtbi.2000.2150.

Viceconti, M., Pappalardo, F., Rodriguez, B. et al. (2021). In silico trials: Verification, validation and uncertainty quantification of predictive models used in the regulatory evaluation of biomedical products. Methods 185, 120-127. https://doi.org/10.1016/j.ymeth.2020.01.011.

Vickers, S. P., Jackson, H. C. and Cheetham, S. C. (2011). The utility of animal models to evaluate novel anti-obesity agents. Br J Pharmacol 164, 1248-1262. https://doi.org/10.1111/j.14765381.2011.01245.x.

Wagner, I., Materne, E. M., Brincker, S. et al. (2013). A dynamic multi-organ-chip for long-term cultivation and substance testing proven by 3D human liver and skin tissue co-culture. Lab Chip 13, 3538-3547. https://doi.org/10.1039/c3lc50234a. 
bioRxiv preprint doi: https://doi.org/10.1101/2021.08.18.456693; this version posted August 19,2021 . The copyright holder for this preprint

(which was not certified by peer review) is the author/funder, who has granted bioRxiv a license to display the preprint in perpetuity. It is made available under aCC-BY-NC-ND 4.0 International license.

Wilson, Z. E., Rostami-Hodjegan, A., Burn, J. L. et al. (2003). Inter-individual variability in levels of human microsomal protein and hepatocellularity per gram of liver. Br J Clin Pharmacol 56, 433-440. https://doi.org/10.1046/j.1365-2125.2003.01881.x.

Wu Jin, P., Rousset, N., Hierlemann, A. et al. (2021). A Microfluidic Hanging-Drop-Based Islet Perifusion System for Studying Glucose-Stimulated Insulin Secretion From Multiple Individual Pancreatic Islets . Front Bioeng Biotechnol 9, 352. Available at: https://www.frontiersin.org/article/10.3389/fbioe.2021.674431.

Yu, J., Cilfone, N. A., Large, E. M. et al. (2015). Quantitative Systems Pharmacology Approaches Applied to Microphysiological Systems (MPS): Data Interpretation and Multi-MPS Integration. CPT Pharmacometrics Syst Pharmacol 4, 585-594. https://doi.org/10.1002/psp4.12010.

Zhang, B., Korolj, A., Lai, B. F. L. et al. (2018). Advances in organ-on-a-chip engineering. Nat Rev Mater 3, 257-278. https://doi.org/10.1038/s41578-018-0034-7. 


\section{Figures}

A

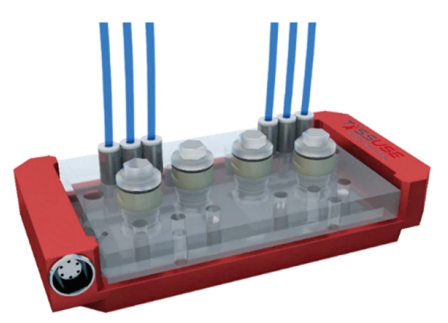

C Three glycemic levels:
Hyperglycemia (11 mM glucose)
B

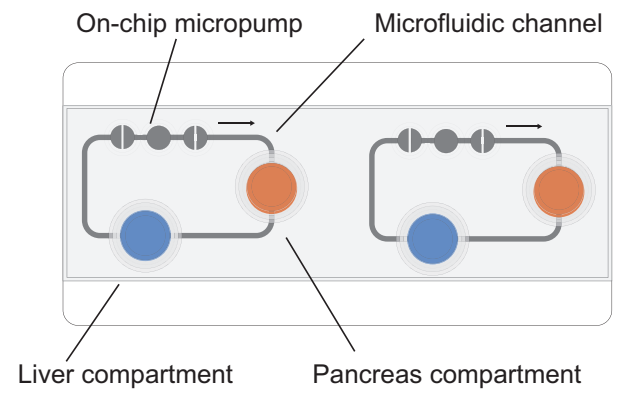

Liver spheroid formation

Islets preculture

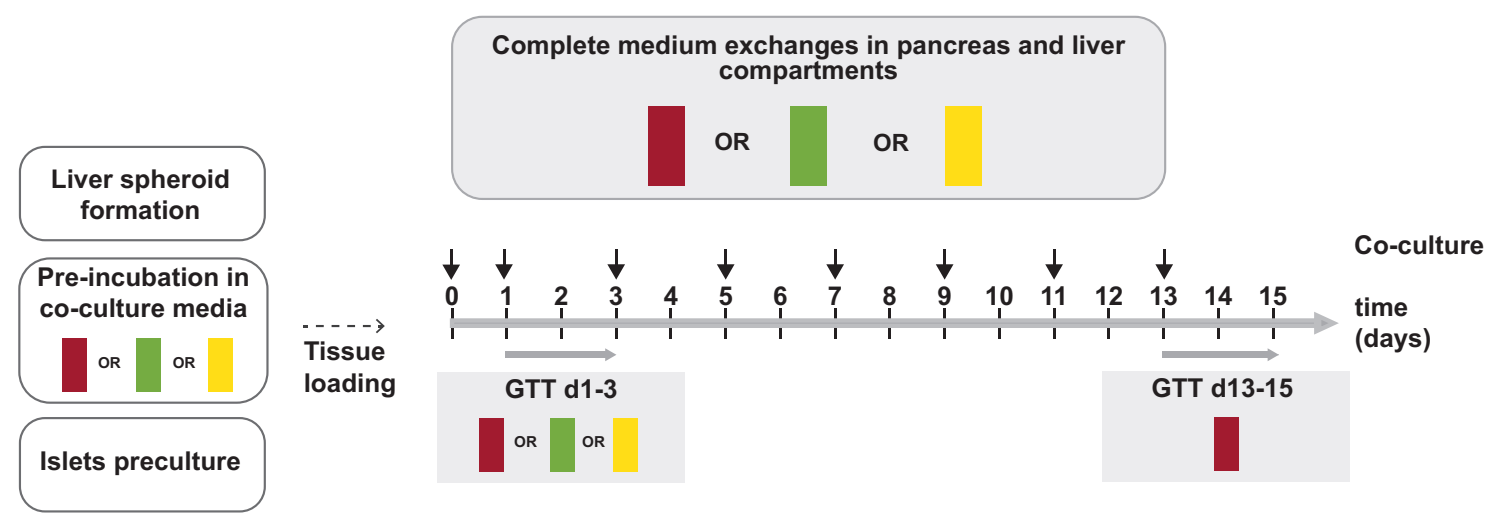

Figure 1: In vitro experiments. (A) A 3D view of the Chip2 (reprinted from (Bauer et al., 2017) with permission under Creative Commons license). (B) Illustration of the Chip2 from underneath, including liver (blue) and pancreas (orange) compartments, the microfluidic channel, and the on-chip micropump. The arrow indicates the direction of flow between culture compartments. (C) Generic study design used in the experiments performed in the study. In five independent MPS experiments ( $\mathrm{N}=5$ ), co-cultures or single organ-cultures were exposed to either hyperglycemia (11 mM glucose, $\mathrm{N}=5$, red), normoglycemia (5.5 mM glucose, $\mathrm{N}=3$, green) or hypoglycemia (2.8 $\mathrm{mM}$ glucose, $\mathrm{N}=1$, yellow). Medium exchanges occurred every $48 \mathrm{~h}$ (vertical black arrows) and glucose tolerance tests (GTTs) were performed on indicated days (horizontal gray arrows). GTT d13-15 was initiated by adding coculture medium with $11 \mathrm{mM}$ glucose into each of the culture compartments. In GTT d1-3, the glucose concentration in the co-culture medium was set to the glycemic level corresponding to each regime (11 mM, 5.5 $\mathrm{mM}$ and $2.8 \mathrm{mM}$ for co-cultures exposed to hyperglycemia, normoglycemia and hypoglycemia, respectively). Samples of the medium were taken $0,8,24$ and $48 \mathrm{~h}$ after the start of the GTT. 


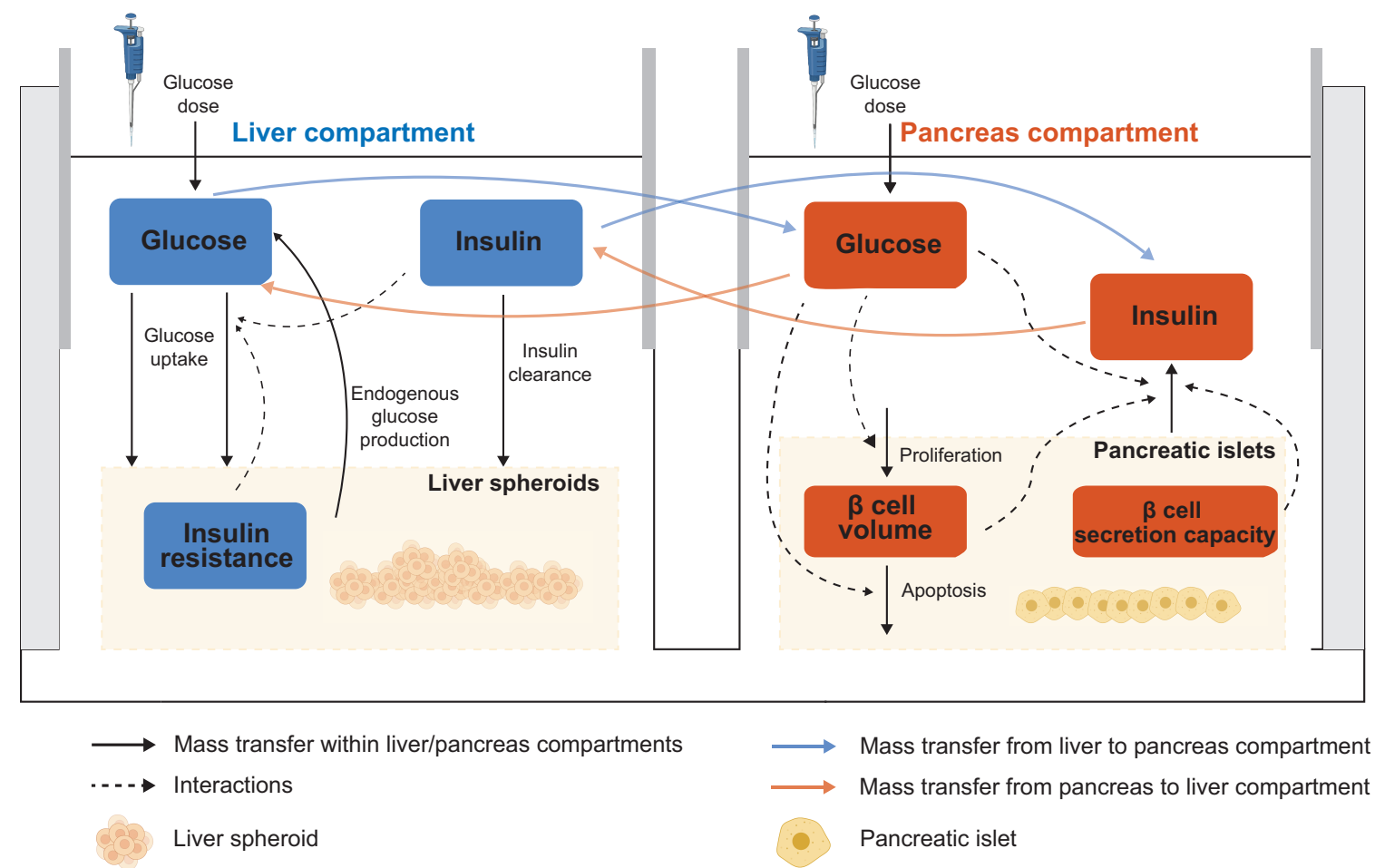

Figure 2: Graphical illustration of the computational model of the liver-islet MPS. The physiological variables described by the model are displayed in blue and red text boxes. The solid arrows represent changes in these variables, mainly because of metabolic fluxes within each organoid compartment (black arrows) or between compartments (blue and orange arrows). Interactions between the variables are represented as dashed arrows. 


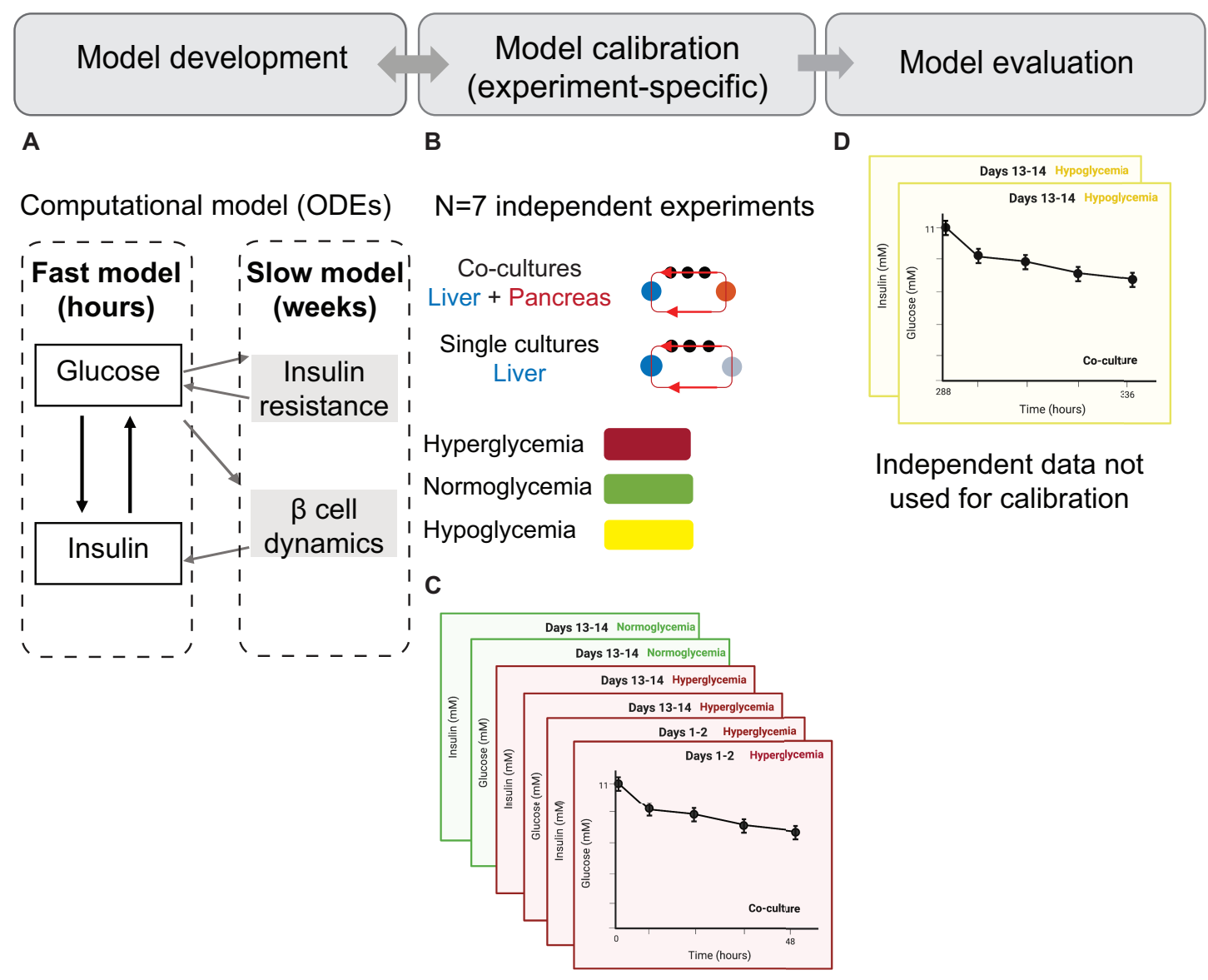

Calibration data

Figure 3: Flow chart illustrating the steps in the modelling approach. (A) Model development: The computational model includes a fast component governing daily interactions between glucose and insulin, and a slow component describing the development of insulin resistance and pancreatic $\beta$-cell compensation. (B) Model calibration: The parameters in the model were estimated individually for each of the seven experiments $(\mathrm{N}=7)$. Experimental data used for calibration $(\mathbf{C})$ includes time-series measurements of glucose and insulin from both co-cultures and single-liver cultures, as well as from co-cultures exposed to different glycemic regimes (hyperand normoglycemia). These measurements were acquired during 48-hour GTTs initiated at day 1 or day 13. (D) Model evaluation: The model was evaluated against independent data not used in the calibration step. This evaluation was performed in two of the seven experiments $(\mathrm{N}=2)$ in which we acquired additional in vitro measurements to compare against the model predictions. 
A

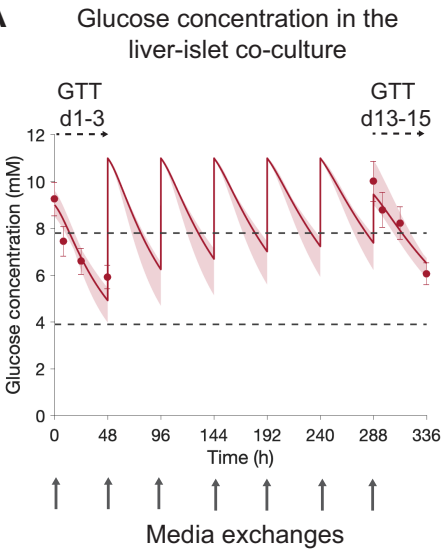

D Integral of excess glucose over time

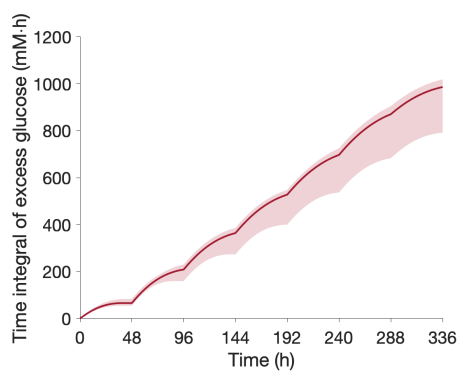

G

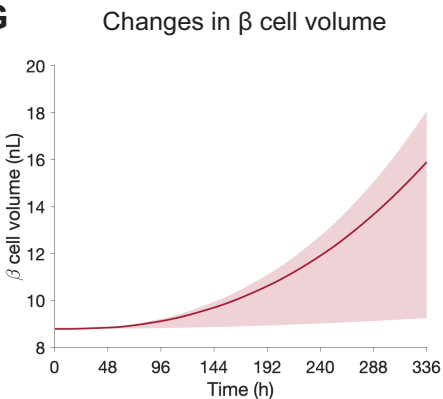

B

Glucose concentration in the single-liver culture
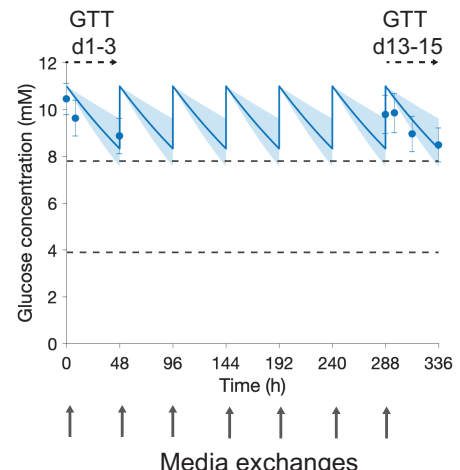

$\mathbf{E}$
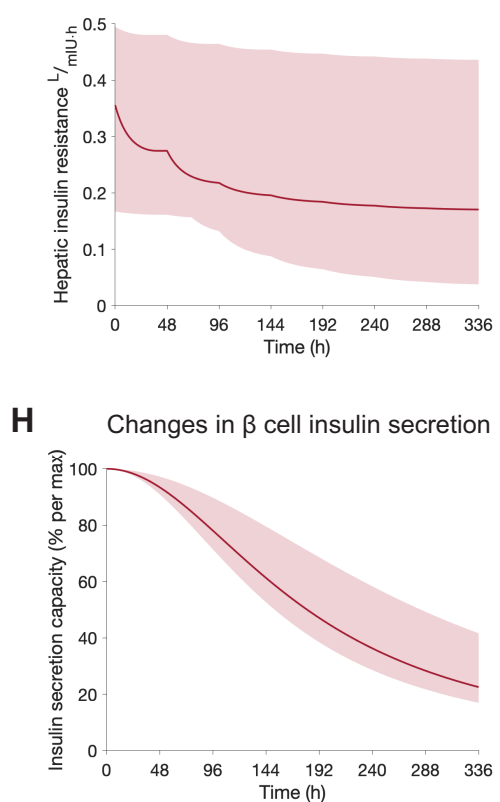

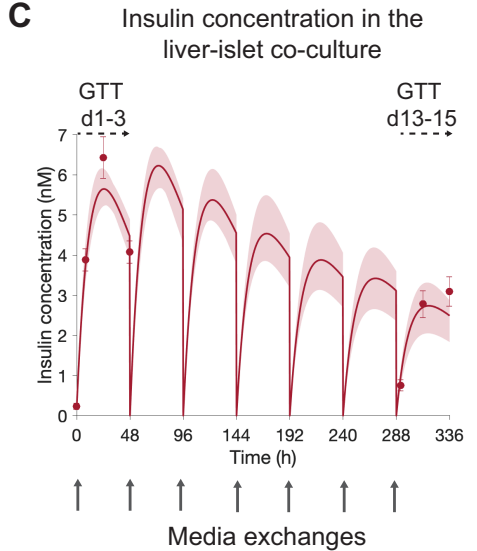

F Daily average glucose concentration

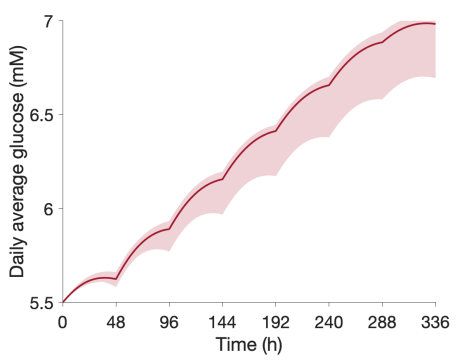

Liver-islet co-culture, $\quad$ Experimental data hyperglycemia Model simulation

Single-liver culture hyperglycemia

Figure 4: Model-based analysis of the physiological processes behind impaired glucose homeostasis in the co-culture. The results correspond to experiment 2 (Bauer et al., 2017). A-C: Comparison between experimental measurements (markers) and model simulations (lines) of glucose concentration in the co-cultures (A), in singleliver cultures (B), and insulin concentration in the liver-islet co-cultures (C). Co-cultures were exposed to hyperglycemic medium (11 mM glucose) at each media exchange (grey arrows). Experimental time-series were acquired during GTTs initiated at day 1 (GTT d1-3) and day 13 (GTT d13-15). The mechanistic variables inferred by the model, which can explain the experimental data in (A-C) are shown in (D-H). (D) Integral of excess glucose (i.e. difference between glucose levels in the co-culture media and $5.5 \mathrm{mM}$ ) over time. This accounts for the effect of exposing the liver spheroids to periods of hyperglycemia during the co-culture time, with the associated decrease in hepatic insulin sensitivity (E) and rise in daily average glucose levels $(\mathbf{F})$. Changes in $\beta$-cell insulin-producing capacity predicted by the model are caused by an increase in pancreatic $\beta$-cell volume $(\mathbf{G})$ and a decay in the individual secretion capacity of $\beta$ cells over time $(\mathbf{H})$. Model uncertainty is depicted as shaded areas in panels AH. Data in panels A-C are presented as mean $\pm \mathrm{SEM}, \mathrm{n}=5$. 
A

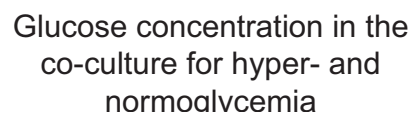

Glucose concentration in the co-culture for hyper- and normoalvcemia

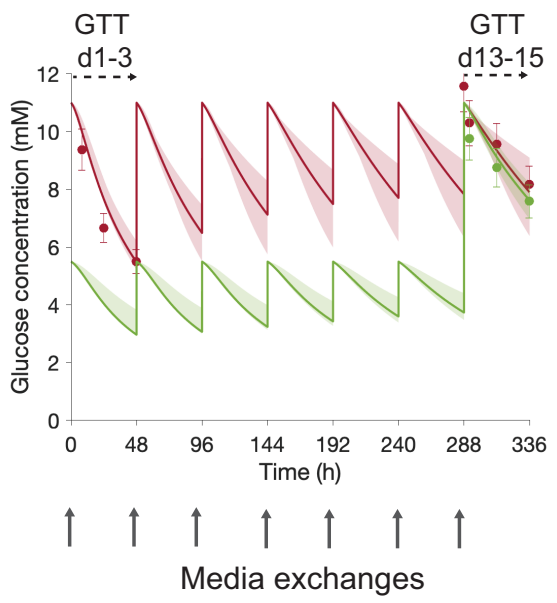

C Hepatic insulin sensitivity

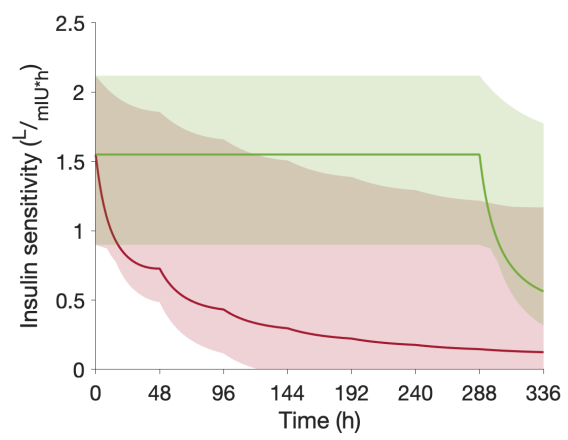

E Changes in $\beta$ cell volume

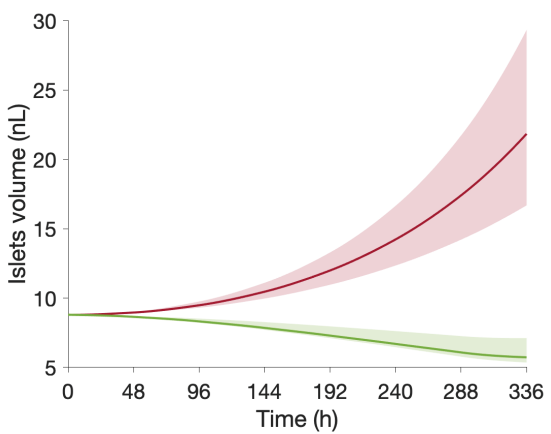

B Insulin concentration in the co-culture for hyper- and normoglycemia

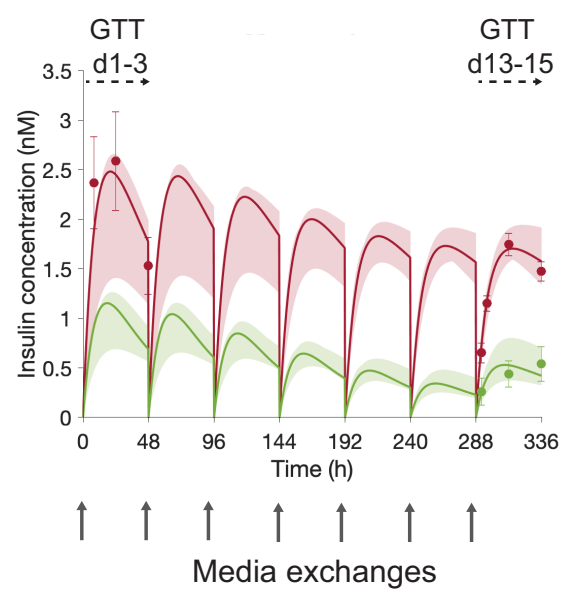

D Daily average glucose concentration

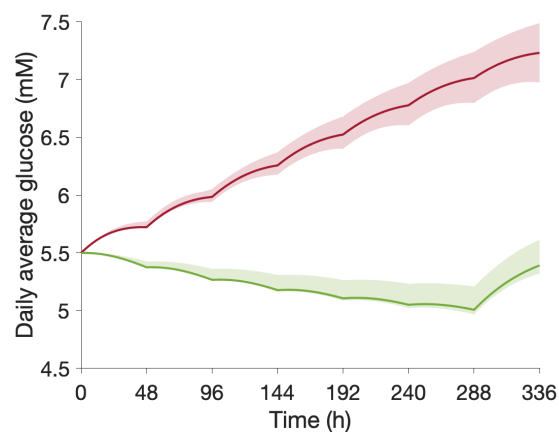

Figure 5: The computational model can explain impaired glucose homeostasis and $\boldsymbol{\beta}$-cell dysfunction under varying glycemic conditions in liver-islet MPS. The results correspond to a single experiment (experiment 3 ). A-B: Comparison between experimental measurements (markers) and model simulations (lines) of glucose concentration (A), and insulin concentration (B) over the 15 days of liver-islet co-culture. Co-cultures were exposed to either a hyperglycemic (11 mM glucose, red) or normoglycemic medium (5.5 mM glucose, green) in each media exchange (grey arrows) between days 1 and 13. Experimental time-series were acquired during GTTs initiated at day 1 (GTT d1-3) in hyperglycemic co-cultures and day 13 (GTT d13-15) in both hyper- and normoglycemic co-cultures. The model predicts decreased insulin sensitivity (i.e. increased insulin resistance) in liver spheroids from hyperglycemic co-cultures, compared to those under normoglycemia (C). The predicted 
differences in daily glucose levels over the co-culture time between both glycemic regimes (D) lead to different trends in $\beta$-cell volume changes (E) due to the bell-shaped relationship between average daily glucose and net $\beta$ cell growth rate in the model. Model uncertainty is depicted as shaded areas in panels A-E. Data in panels A-B are presented as mean \pm SEM, $\mathrm{n}=5$. 
A

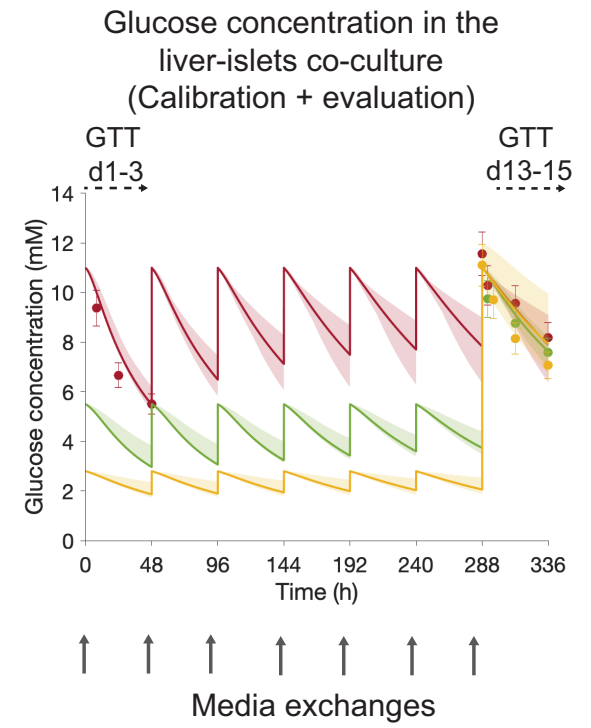

C

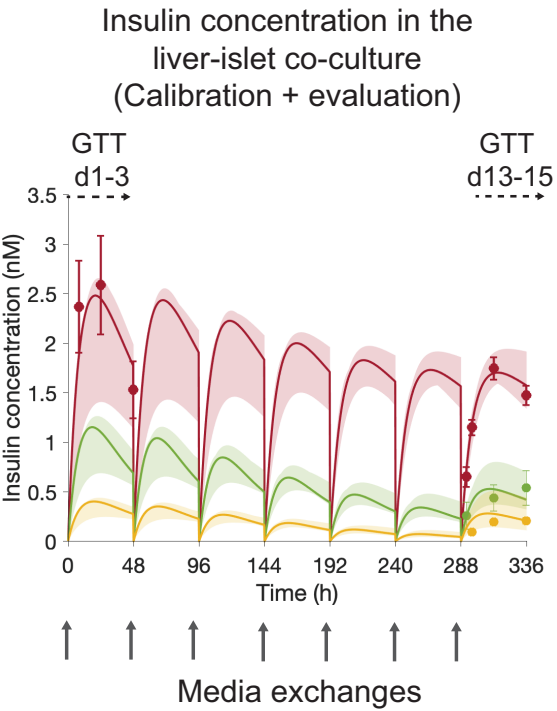

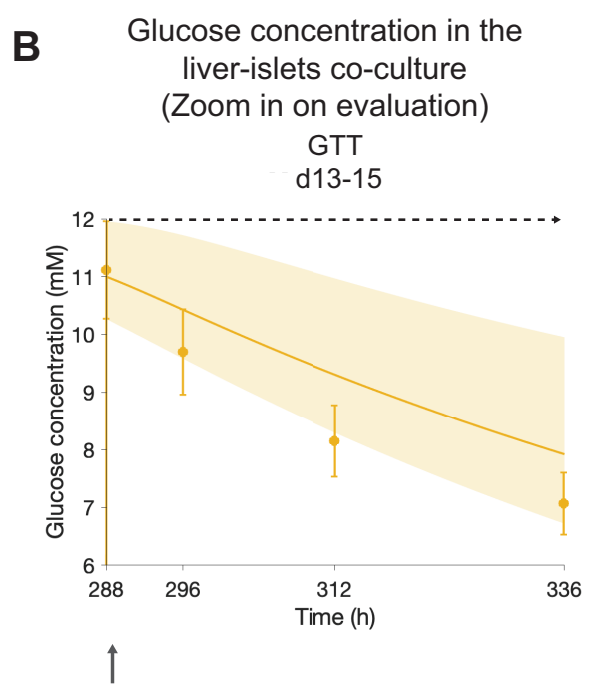

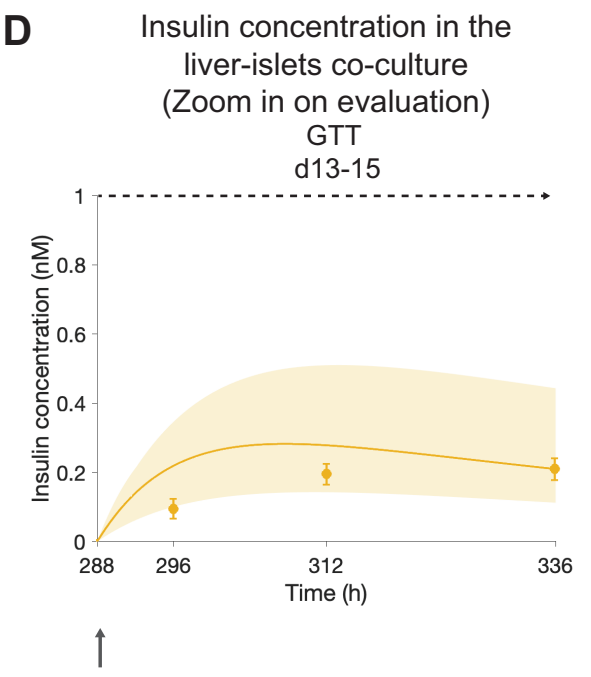

\begin{tabular}{|l}
\hline $\begin{array}{l}\text { Liver-islet co-culture, } \\
\text { hyperglycemia (calibration) }\end{array}$ \\
$\begin{array}{l}\text { Liver-islet co-culture, } \\
\text { normoglycemia (calibration) }\end{array}$ \\
$\begin{array}{l}\text { Liver-islet co-culture, } \\
\text { hypoglycemia (prediction) }\end{array}$ \\
\hline
\end{tabular}

Figure 6: The model can predict GTT responses in the end of the liver-islet co-culture following repeated exposure to hypoglycemic media concentrations. The results correspond to a single experiment (experiment 3 ). In $\mathbf{A , C}$, experimental measurements of glucose and insulin concentrations (markers) are compared to model simulations for hyper- and normo-glycemia (red and green, lines) and model predictions for hypoglycemia (yellow lines) over the 15 days of co-culture. Co-cultures were exposed to either a hyperglycemic (11 mM glucose, red), normoglycemic (5.5 mM glucose, green) and hypoglycemic (2.8 $\mathrm{mM}$ glucose, yellow) media in each media exchange (grey arrows) between days 1 and 13. Experimental time-series were acquired during GTTs initiated at day 1 (GTT d1-3) in hyperglycemic co-cultures and day 13 (GTT d13-15) in all co-cultures (hyper-, normo- and hypoglycemic). For a clearer comparison between the model predictions and the corresponding experimental data 
bioRxiv preprint doi: https://doi.org/10.1101/2021.08.18.456693; this version posted August 19,2021 . The copyright holder for this preprint

(which was not certified by peer review) is the author/funder, who has granted bioRxiv a license to display the preprint in perpetuity. It is made available under aCC-BY-NC-ND 4.0 International license.

for the evaluation part, panels B and D zoom in on the GTT performed at day 13 for the hypoglycemic arm glucose (B) and insulin (D). Model uncertainty is depicted as shaded areas in A-D. Data in panels A,C are presented as mean \pm SEM, $n=5$. 


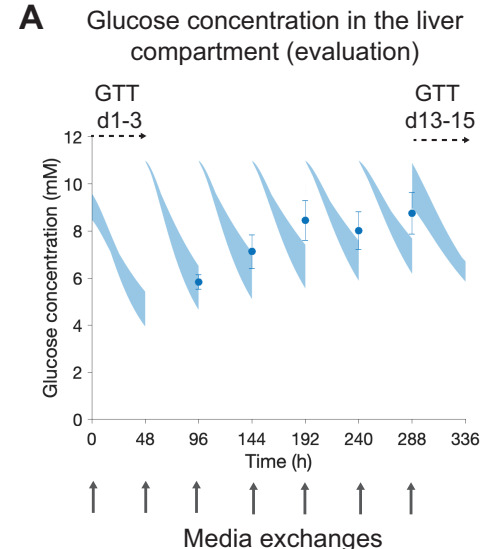

D Insulin concentration in the liver compartment (evaluation)

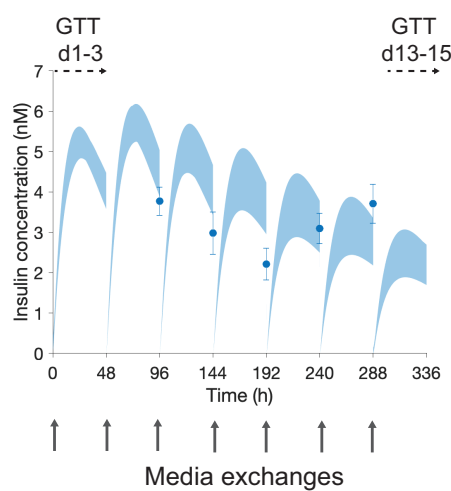

B

Glucose concentration in the pancreas compartment (evaluation)

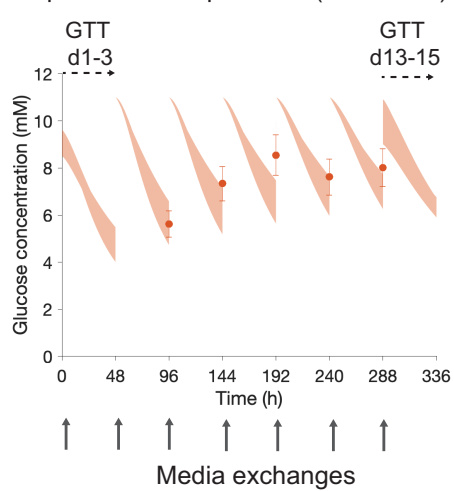

E Insulin concentration in the pancreas compartment (evaluation)

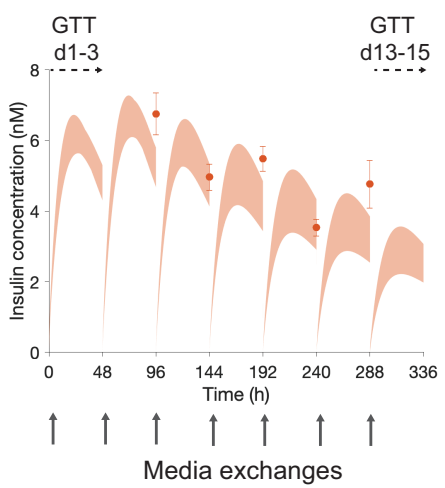

C Measured glucose concentration in the liver-islet co-culture (calibration)

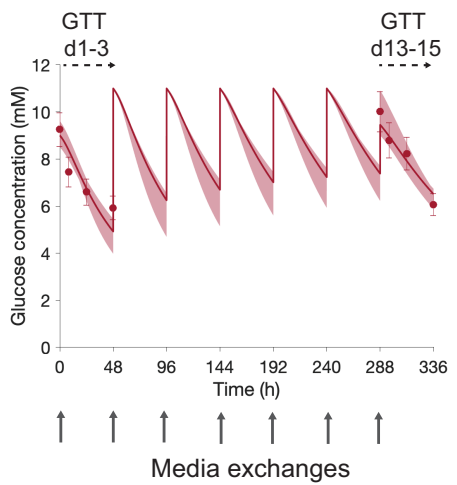

F Measured insulin concentration in the liver-islet co-culture (calibration)

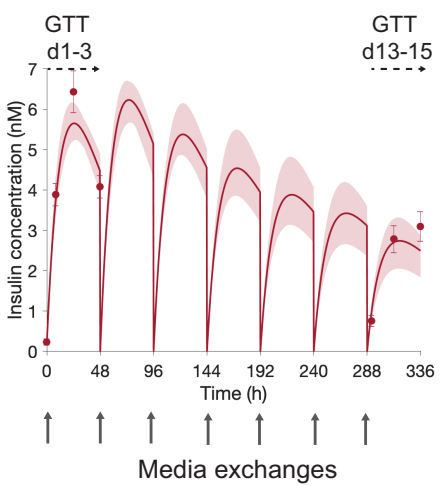

Figure 7: Model predictions of glucose and insulin concentrations in each organ compartment. The results correspond to a single experiment (experiment 2). A,B Comparison between model predictions of glucose in the liver and islets compartments, respectively, and the corresponding experimental data (markers). D,E Comparison between model predictions of insulin in the liver and islets compartments, respectively, and the corresponding experimental data (markers). Co-cultures were exposed to hyperglycemic medium (11 mM glucose) in each media exchange (grey arrows). The calibration data $(\mathbf{C , F})$ consisted of glucose and insulin measurements acquired during GTTs initiated at day 1 (GTT d1-3) and day 13 (GTT d13-15). In the calibration data (C,F), glucose and insulin concentrations were measured by pooling samples of $15 \mu \mathrm{L}$ from each compartment (i.e. measuring the average concentration of the two compartments), while concentrations in the evaluation dataset $(\mathbf{A}, \mathbf{B}, \mathbf{D}, \mathbf{E})$ were measured independently in each culture compartment (liver or pancreas). Data points for evaluation were acquired 48 hours following each media exchange between days 3 and 13. Model uncertainty is depicted as shaded areas in (A-F). Data are presented as mean $\pm \mathrm{SEM}, \mathrm{n}=10$. 
A

Glucose concentration in the liver compartment after translation to human

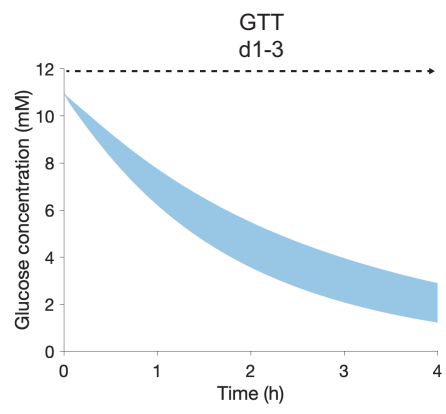

D Insulin concentration in the liver compartment after translation to human

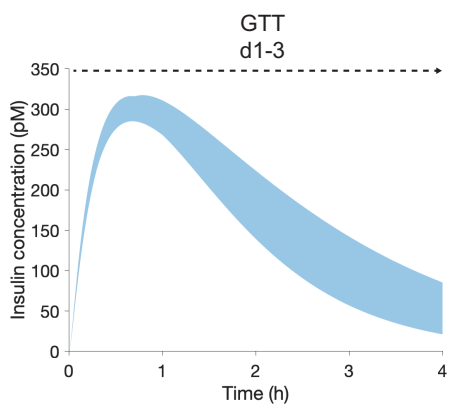

B Glucose concentration in the pancreas compartment after translation to human

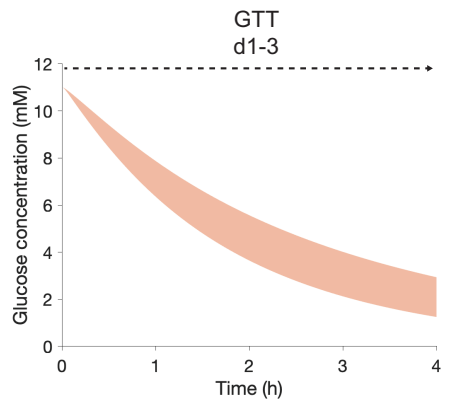

E

Insulin concentration in the pancreas compartment after translation to human

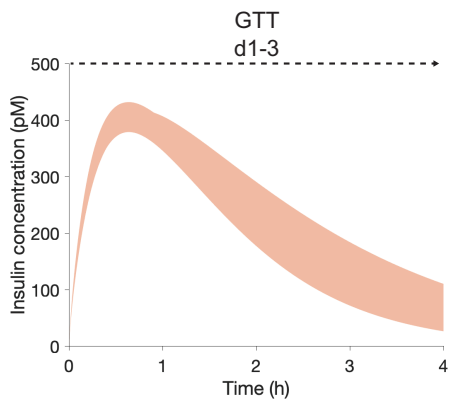

C

Glucose concentration in the liver-islet co-culture after translation to human

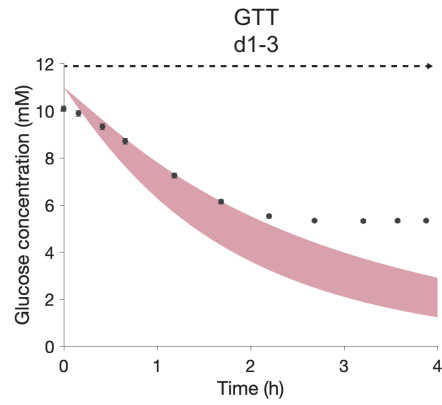

F Insulin concentration in the liver-islet co-culture after translation to human

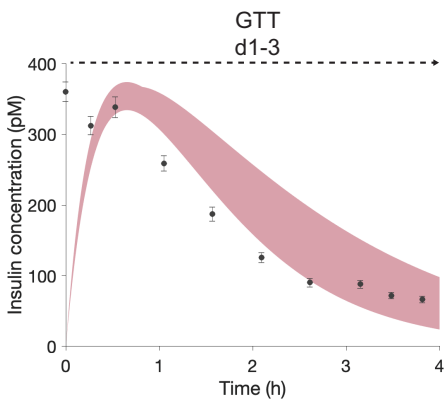

Liver-islet co-culture, liver compartment,

after translation to human

Liver-islet co-culture, pancreas compartment, after translation to human

- Liver-islet co-culture,measured (average), after translation to human
Experimental data meal response in humans

Model uncertainty

Figure 8: Model-based extrapolation of glucose and insulin concentrations in the liver-islet MPS to human meal responses. The results correspond to a single experiment (experiment 1). A,B,D,E Model predictions of glucose $(\mathbf{A}, \mathbf{B})$ and insulin $(\mathbf{D}, \mathbf{E})$ in the liver and islets compartments after scaling to human. $\mathbf{C}$ shows the comparison between the model prediction of plasma glucose concentration in a corresponding human and experimental data of glucose response to a meal in healthy subjects (Man et al., 2007). The model-based prediction of the insulin response and the experimental measurements of insulin are compared in $\mathbf{F}$. The predictions are computed for the GTT initiated at day 1 (GTT d1-3). The experimental data, reported in a previous study by Dalla Man et al. (Man et al., 2007), were acquired in a group of 204 normal subjects. We consider the time point of peak glucose concentration in the experimental data as time $=0 \mathrm{~h}$ for this study, since the liver-pancreas MPS lacks an intestinal compartment and glucose is administered directly to the liver and pancreas compartments. Data are presented as mean \pm SEM, $n=204$. Model uncertainty is depicted as shaded areas in (A-F). 


\section{Tables}

Table 1: Parameters in the computational model. Parameters specified as constant were not included in the parameter estimation routine. The estimated parameter values for each MPS experiment are listed in Supplementary Table S3.

Parameter Description Unit Estimation/reference

\begin{tabular}{|c|c|c|c|}
\hline \multicolumn{4}{|c|}{ Operating conditions } \\
\hline$V_{m, l i v e r}$ & $\begin{array}{l}\text { Volume of co-culture medium in the } \\
\text { liver compartment }\end{array}$ & $\mathrm{L}$ & $\begin{array}{l}\text { Set based on MPS operating } \\
\text { conditions }\left(3 \cdot 10^{-4} \text {, constant }\right)\end{array}$ \\
\hline$V_{m, \text { pancreas }}$ & $\begin{array}{l}\text { Volume of co-culture medium in the } \\
\text { pancreas compartment }\end{array}$ & $\mathrm{L}$ & $\begin{array}{l}\text { Set based on MPS operating } \\
\text { conditions }\left(3 \cdot 10^{-4} \text {, constant }\right)\end{array}$ \\
\hline$V_{\text {HepaRG }, \text { spheroids }}$ & $\begin{array}{l}\text { Total volume of HepaRG cells in the } \\
\text { MPS }\end{array}$ & $\mathrm{L}$ & $\begin{array}{l}\text { Set based on MPS operating } \\
\text { conditions }\left(3.4 \cdot 10^{-6}, \text { constant }\right)\end{array}$ \\
\hline$V_{\text {sample,liver }}$ & $\begin{array}{l}\text { Volume of co-culture medium collected } \\
\text { from the liver compartment in each } \\
\text { sample }\end{array}$ & $\mathrm{L}$ & $\begin{array}{l}\text { Set based on MPS operating } \\
\text { conditions }\left(1.5 \cdot 10^{-5}, \text { constant }\right)\end{array}$ \\
\hline$V_{\text {sample,pancreas }}$ & $\begin{array}{l}\text { Volume of co-culture medium collected } \\
\text { from the pancreas compartment in each } \\
\text { sample }\end{array}$ & $\mathrm{L}$ & $\begin{array}{l}\text { Set based on MPS operating } \\
\text { conditions }\left(1.5 \cdot 10^{-5} \text {, constant }\right)\end{array}$ \\
\hline$Q$ & Flow rate between culture compartments & $\mathrm{L} / \mathrm{h}$ & $\begin{array}{l}\text { Set based on MPS operating } \\
\text { conditions }\left(2.96 \cdot 10^{-4} \text {, constant }\right)\end{array}$ \\
\hline \multicolumn{4}{|l|}{ Liver spheroids } \\
\hline$E_{G 0}$ & $\begin{array}{l}\text { Insulin-independent glucose disposal } \\
\text { rate }\end{array}$ & $1 / \mathrm{h}$ & Estimated from data \\
\hline$C L_{I, \text { spheroids }}$ & Insulin clearance & $1 / \mathrm{h}$ & Estimated from data \\
\hline \multicolumn{4}{|c|}{ Insulin resistance (slow model) } \\
\hline$G_{\text {normo }}$ & $\begin{array}{l}\text { Glucose concentration for } \\
\text { normoglycemia }\end{array}$ & $\mathrm{mmol} / \mathrm{L}$ & $\begin{array}{l}\text { Set based on physiological } \\
\text { considerations }(5.5, \text { constant })\end{array}$ \\
\hline$S_{I 0}$ & $\begin{array}{l}\text { Insulin sensitivity at the start of the co- } \\
\text { culture }\end{array}$ & $\mathrm{L} / \mathrm{mIU} / \mathrm{h}$ & Estimated from data \\
\hline$I_{\max , S i}$ & $\begin{array}{l}\text { Maximal fractional reduction of insulin } \\
\text { sensitivity }\end{array}$ & & Estimated from data \\
\hline$E C 50_{S i}$ & $\begin{array}{l}\text { Value of time integral of excess glucose } \\
\text { providing half of the maximal fractional } \\
\text { reduction. }\end{array}$ & $\mathrm{mmol} \cdot \mathrm{h} / \mathrm{L}$ & Estimated from data \\
\hline
\end{tabular}

\begin{tabular}{llll}
\hline Pancreatic islets & & & \\
\hline$\sigma_{\text {max }}$ & $\begin{array}{l}\text { Insulin secretion rate of the beta cells at } \\
\text { the start of the co-culture } \\
\text { Parameter defining the sigmoidal } \\
\text { dependence of the insulin secretion } \\
\text { capacity on time }\end{array}$ & $\mathrm{mIU} / \mathrm{L} / \mathrm{h}$ & Estimated from data \\
$\begin{array}{l}\text { Glucose concentration resulting in half- } \\
\text { of-maximum response to insulin of the } \beta \\
\text { cells }\end{array}$ & $\mathrm{mmol} / \mathrm{L}$ & $\begin{array}{l}\text { From literature (Topp et al., } \\
\text { 2000) (7.86, constant) }\end{array}$ \\
\hline$\beta$ cell dynamics $($ slow model) & Rate of $\beta$-cell death at zero glucose & $1 / \mathrm{h}$ & $\begin{array}{l}\text { From literature (Topp et al., } \\
\text { 2000) }\left(2.5 \cdot 10^{-3}, \text { constant) }\right.\end{array}$
\end{tabular}




$r_{1}$
$r_{2}$
$k_{v}$
$\tau_{\text {slow }}$

Rate constant that determines the dependence of the replication and apoptosis rates on glucose Rate constant that determines the dependence of the replication and apoptosis rates on glucose of $\beta$-cell number

$\tau_{\text {slow }}$ Constant for time averaging of glucose Scaling parameter for the rate of change concentration

$$
\begin{array}{ll}
\mathrm{L} / \mathrm{mmol} / \mathrm{h} & \text { From literature (Topp et al., } \\
& \text { 2000) }\left(6.3 \cdot 10^{-4}\right. \text {, constant) } \\
\mathrm{L}^{2} / \mathrm{mmol}^{2} / & \text { From literature (Topp et al., } \\
\mathrm{h} & \text { 2000) }\left(3.24 \cdot 10^{-5},\right. \text { constant) }
\end{array}
$$

Estimated from data

$\mathrm{h}$

Estimated from literature

(Chick, 1973; Swenne, 1982;

Bonner-Weir et al., 1989;

Bernard et al., 1998; Paris et al., 2003) (500, constant)

\section{Experimental errors}

$\Delta G_{d 1} \quad$ Offset in glucose concentration related

$\mathrm{mmol} / \mathrm{L} \quad$ Estimated from data

to co-culture media exchange in the

GTT initiated at day 1 (GTT d1-3)

$\Delta G_{d 13}$

Offset in glucose concentration related

$\mathrm{mmol} / \mathrm{L}$

Estimated from data to co-culture media exchange in the GTT initiated at day 13 (GTT d13-15)

$\Delta I_{d 1}$ Offset in insulin concentration related to $\mathrm{mIU} / \mathrm{L}$

Estimated from data co-culture media exchange in in the GTT initiated at day 1 (GTT d1-3)

$\Delta I_{d 13}$ Offset in insulin concentration related to co-culture media exchange in in the $\mathrm{mIU} / \mathrm{L}$ Estimated from data GTT initiated at day 13 (GTT d13-15) 
Table 2: Extrapolation of parameter values in the computational model to perform in vitro to in vivo translation. The results correspond to experiment 1 .

\begin{tabular}{|c|c|c|c|}
\hline \multirow[b]{2}{*}{ Parameter } & \multirow[b]{2}{*}{ Description (units) } & \multicolumn{2}{|r|}{ Value } \\
\hline & & $\begin{array}{l}\text { In vitro } \\
\text { (MPS) }\end{array}$ & $\begin{array}{l}\text { Translation to in } \\
\text { vivo (human) }\end{array}$ \\
\hline$V_{\text {HepaRG,spheroids }}$ & Total volume of HepaRG cells (L) & $3.4 \cdot 10^{-6}$ & 0.34 \\
\hline$V_{\beta, \text { islets }}(0)$ & $\begin{array}{l}\text { Total volume of pancreatic } \beta \text { cells at the } \\
\text { beginning of the co-culture (L) }\end{array}$ & $8.8 \cdot 10^{-9}$ & $8.8 \cdot 10^{-4}$ \\
\hline$V_{m, l i v e r}$ & $\begin{array}{l}\text { Volume of co-culture medium in the liver } \\
\text { compartment (L) }\end{array}$ & $3 \cdot 10^{-4}$ & 1.5 \\
\hline$V_{m, i s l e t s}$ & $\begin{array}{l}\text { Volume of co-culture medium in the islets } \\
\text { compartment (L) }\end{array}$ & $3 \cdot 10^{-4}$ & 1.5 \\
\hline Q & $\begin{array}{l}\text { Flow rate between culture compartments } \\
(\mathrm{L} / \mathrm{h})\end{array}$ & $2.96 \cdot 10^{-4}$ & 35.5 \\
\hline$E_{G 0}$ & $\begin{array}{l}\text { Hepatic insulin-independent glucose disposal } \\
\text { rate }(\mathrm{mmol} / \mathrm{L} / \mathrm{h})\end{array}$ & 1.47 & 3.25 \\
\hline$S_{I 0}$ & $\begin{array}{l}\text { Hepatic insulin sensitivity at the start of the } \\
\text { co-culture }(\mathrm{L} / \mathrm{mIU} / \mathrm{h})\end{array}$ & $5 \cdot 10^{-3}$ & $1.1 \cdot 10^{-2}$ \\
\hline$\sigma_{\max }$ & $\begin{array}{l}\text { Maximal insulin secretion rate per unit } \\
\text { volume of } \beta \text { cells }(\mathrm{mIU} / \mathrm{L} / \mathrm{h})\end{array}$ & $6 \cdot 10^{6}$ & $10^{6}$ \\
\hline$C L_{i, h e p}$ & Hepatic insulin clearance $(1 / \mathrm{h})$ & 17.81 & 35.62 \\
\hline
\end{tabular}

